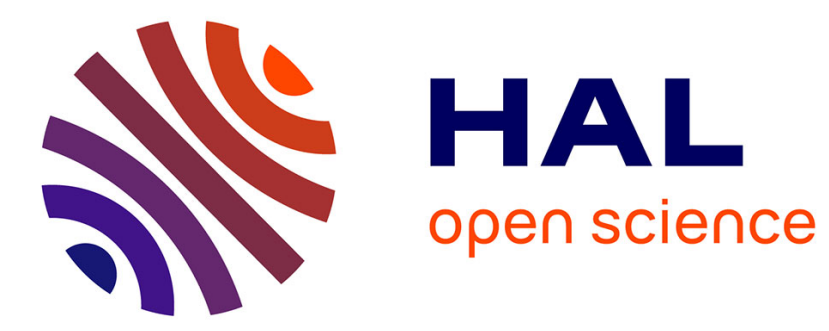

\title{
A stochastic maximum principle for a stochastic differential game of a mean-field type
}

John J. A. Hosking

\section{To cite this version:}

John J. A. Hosking. A stochastic maximum principle for a stochastic differential game of a mean-field type. 2011. hal-00641090v2

\section{HAL Id: hal-00641090 \\ https://hal.inria.fr/hal-00641090v2}

Preprint submitted on 15 Nov 2011

HAL is a multi-disciplinary open access archive for the deposit and dissemination of scientific research documents, whether they are published or not. The documents may come from teaching and research institutions in France or abroad, or from public or private research centers.
L'archive ouverte pluridisciplinaire HAL, est destinée au dépôt et à la diffusion de documents scientifiques de niveau recherche, publiés ou non, émanant des établissements d'enseignement et de recherche français ou étrangers, des laboratoires publics ou privés. 


\title{
A stochastic maximum principle for a stochastic differential game of a mean-field type*
}

\author{
John J. A. Hosking ${ }^{\dagger}$
}

November 15, 2011

\begin{abstract}
We construct a stochastic maximum principle (SMP) which provides necessary conditions for the existence of Nash equilibria in a certain form of $N$-agent stochastic differential game (SDG) of a meanfield type. The information structure considered for the SDG is of a possible asymmetric and partial type. To prove our SMP we use a spike-variation approach with adjoint representation techniques, analogous to that of S. Peng in the optimal stochastic control context. In our proof we apply adjoint representation procedures at three points. The first-order adjoint processes are defined as solutions to certain mean-field backward stochastic differential equations, and second-order adjoint processes of a first type are defined as solutions to certain backward stochastic differential equations. Secondorder adjoint processes of a second type are defined as solutions of backward stochastic equations of a type that we introduce in this paper, and which we term conditional mean-field backward stochastic differential equations. From the resulting representations, we show that the terms relating to these second-order adjoint processes of the second type are of an order such that they do not appear in our final SMP equations.
\end{abstract}

Keywords: Stochastic maximum principle, stochastic differential games, Nash equilibria, meanfield type stochastic equations, mean-field type backward stochastic equations.

American Mathematical Society, 2010 Mathematics Subject Classification: 93E99, 60H99, 91A06, 91A15.

\section{Introduction}

In this paper we construct a stochastic maximum principle (SMP) which provides necessary conditions for the existence of Nash equilibria in a certain $N$-agent stochastic differential game (SDG) of a meanfield type. The exact description of this form of $N$-agent SDG is presented in Section 2; in summary, it is a SDG with:

- a state process of the form

$$
\begin{aligned}
X^{(u)}(\omega, t) & =x_{0}+\int_{0}^{t} b\left(\omega, s, X^{(u)}(\omega, s), \int_{\Omega} \phi_{b}\left(\omega^{\prime}, s, X^{(u)}\left(\omega^{\prime}, s\right), u\left(\omega^{\prime}, s\right)\right) \mathbb{P}\left(\mathrm{d} \omega^{\prime}\right), u(\omega, s)\right) \mathrm{d} s \\
+ & \int_{0}^{t} \sigma\left(\omega, s, X^{(u)}(\omega, s), \int_{\Omega} \phi_{\sigma}\left(\omega^{\prime}, s, X^{(u)}\left(\omega^{\prime}, s\right), u\left(\omega^{\prime}, s\right)\right) \mathbb{P}\left(\mathrm{d} \omega^{\prime}\right), u(\omega, s)\right) \mathrm{d} B(\omega, s)
\end{aligned}
$$

for each $u=\left(u_{1}, \ldots, u_{N}\right)$ comprising of each agent's employed admissible control process; and

- performance functionals $\left\{J_{i} \mid i \in\{1, \ldots, N\}\right\}$ of the form

$$
\begin{array}{r}
J_{i}(u):=\int_{\Omega}\left[\int_{0}^{T} f_{i}\left(\omega, s, X^{(u)}(\omega, s), \int_{\Omega} \phi_{f_{i}}\left(\omega^{\prime}, s, X^{(u)}\left(\omega^{\prime}, s\right), u\left(\omega^{\prime}, s\right)\right) \mathbb{P}\left(\mathrm{d} \omega^{\prime}\right), u(\omega, s)\right) \mathrm{d} s\right. \\
\left.+g_{i}\left(\omega, X^{(u)}(\omega, T), \int_{\Omega} \phi_{g_{i}}\left(\omega^{\prime}, X^{(u)}\left(\omega^{\prime}, T\right)\right) \mathbb{P}\left(\mathrm{d} \omega^{\prime}\right)\right)\right] \mathbb{P}(\mathrm{d} \omega) .
\end{array}
$$

*A version of this paper which addresses a multidimensional form of the problem that is considered here, has been deposited on HAL - INRIA (see [10])

$\dagger$ INRIA Paris - Rocquencourt, Équipe - projet MATHFI, Domaine de Voluceau, BP 105, 78153 Le Chesnay Cedex, France (John.Hosking@inria.fr) 
Key features of the problem we address are that:

- The setting is in general non-Markovian.

- The information structure is in general of an asymmetric and partial type, that is:

- For each agent, their class of admissible controls is a class of predictable processes with respect to a given agent-specific filtration $\mathbb{G}_{i}$ (which are not necessarily equal to one another).

- All of the agent's filtrations, $\left\{\mathbb{G}_{i} \mid i \in\{1, \ldots, N\}\right\}$, are subfiltrations of a reference filtration $\mathbb{F}$.

- For each agent, their admissible control processes are valued in a given agent-specific complete separable metric space - which is not necessarily convex.

We construct our SMP using a spike variation approach with adjoint representation techniques, analogous to that of Peng [21] where a SMP is constructed for a certain form of stochastic optimal control problem in a non-mean-field setting. The approach we follow here is also analogous, in certain respects, to that in the works of [19] and [5], in which the original work of Peng [21] is further developed in the directions of stochastic control problems with multidimensional performance functionals (including the issue of Nash equilibria in SDGs), and a mean-field type stochastic optimal control problem, respectively.

In [19] the authors develop an approach that is to some extent different to the approach of Peng [21], and they allow for the case of multidimensional performance functionals as well as for classes of admissible control processes that satisfy a weaker integrability condition then that stated in [21]. A SMP providing necessary conditions for Nash equilibria in a certain N-agent SDG-with a standard information structure and not of a mean-field type - is presented in Theorem 5.2 of [19].

In [5] a Peng-type SMP is constructed for a certain stochastic optimal control problem of a meanfield type. The generality of the SDG problem that we consider in this paper and that of the stochastic optimal control problem considered in [5] is different. In particular, we allow for the possibility of an asymmetric and partial information structure for our SDG. Moreover, a fundamental difference between these two works is the manner in which the limits relating to the respective second form of quadratic-type terms, as we refer to them (see the proof of Theorem 3.6 for further explanation), are calculated. In [5] the relevant limit is implied to be zero by establishing an appropriate upper bound for the modulus of those terms. In this work we calculate the relevant limit by applying the adjoint representation procedure a third time, defining the relevant adjoint processes as solutions to a backward stochastic equation of a type that we introduce in this paper, and which we term condition mean-field backward stochastic differential equations (CMFBSDEs). We propose that our approach to deal with the second form of quadratic-type terms is no more complicated than that of [5]. Since, having introduced the concept of CMFBSDEs, then our approach to dealing with these terms is essentially the application of some of the same general ideas, such as the adjoint representation techniques, from [21], which we also make use of in treating, what we refer to as, the linear-type terms and the first form of quadratic-type terms in our problem here. Also, it would appear that our approach may - in principle - be suitable for further development in an attempt to calculate higherorder expansion terms for the second form of quadratic-terms, which would be of interest in the possible construction of forms of higher-order SMPs.

As is suggested by the form of the state process $X^{(u)}$, and of the performance functionals $\left\{J_{i} \mid i \in\right.$ $\{1, \ldots, N\}\}$, stated above, the term mean-field is broadly used here in the sense which takes:

- a stochastic process of a mean-field type to be a form of stochastic process whose dynamics are a function of its time-marginal probability laws;

- a stochastic control problem of a mean-field type, or a SDG of a mean-field type, to be a stochastic control problem or a SDG where the state process is of a mean-field type (in the above sense) and/or where the performance functionals are of a mean-field type - in the sense that: for a performance functional given as an expectation of the sum of a running performance term and a terminal performance term, then either of these two terms are themselves functions of the timemarginal probability laws of the state process (and not both in a manner that can be reduced to the standard case).

This use of the term mean-field is collectively motivated by its uses in, for example, [7], [6], [5], [1] and $[18]$.

A notion of a mean-field backward stochastic differential equation (MFBSDE) appears in [6] and [7]: this notion of a MFBSDE is essentially a generalization of that of a BSDE which allows the generator term to be a function of the time-marginal probability laws of the solution. Related to the subject of SDEs of a mean-field type, in this sense, is a large literature on approximations by interacting 
particle systems, see for example [17], [4], and [11]. For an example of a stochastic control problem with McKean-Vlasov type SDEs, see [3].

In [1] a SMP is constructed for a mean-field stochastic control problem where both the state dynamics and the performance functionals are of a mean-field type. A fundamental difference between [1] and both this work as well as [5], is that in [1] the construction of their SMP is carried out by an extension of the Bensoussan [2] approach to a mean-field setting; whereas in this paper, and in [5], the SMPs are constructed by extending the Peng [21] approach to a mean-field setting (although with the differences mentioned above). That is, the setting in [1] allows for the admissible controls $u$ to be perturbed in the manner $u+\epsilon \bar{u}$, given some admissible control $\bar{u}$. Our problem of interest is not to permit this form of perturbation; we do not assume the required structure on the range of the control processes for this form of perturbation to be valid, and instead we work with perturbations given by spike-variations. Also, note that in this paper we allow the controls to appear directly - that is other than just via their effect on the state process itself - in the mean-field components of the state process and performance functionals: which is not the case for the control processes in [1] or in [5]. That is, in a certain form, the dynamics and performance functionals in our SDG may be functions of the time-marginal joint probability laws between the state process and the control processes.

Another relevant work is that of [18] which constructs, via results from Malliavin calculus, a SMP for a stochastic control problem in which mean-field terms appear in the considered form of performance functional but not in the dynamics of the state process (which is a controlled Itô-Lévy SDE). By considering a certain form of Bensoussan-type perturbation, the authors make use of the relevant Malliavin calculus duality relations to construct a SMP in terms of the Malliavin derivative and difference operators.

Other work concerned with a notion of mean-field in the area of optimal control and game theory is that of the series of work by J.-M. Lasry and P.-L. Lions, for example [14], [15] and [16]. See also [9]. Mean-field games are the subject of, for example, Section 2 of [16] where a certain form of $N$-agent SDG (with non-mean-field state process and performance functionals, in the sense from before) and certain corresponding equations for the existence of Nash equilibria are considered as the number of agents $N$ tends to infinity. At first sight this form of problem appears to be conceptually different to the problem we consider here, the later being a finite-agent SDG with a mean-field type state process and performance functionals. However, it could be a topic for future research to consider whether there exists some form of relationship between mean-field SDGs of the type addressed here, or some variant of them, and a general notion of mean-field games.

This paper is structured as follows: Section 2 specifies the form of the SDG that we consider, presenting the definitions of the admissible control classes, the state dynamics, and the performance functionals; Section 3 presents the main result of the paper, Theorem 3.6, which presents our SMP for Nash equilibria in our considered SDG; Section 4 provides an existence and uniqueness result for solutions to certain CMFBSDEs; Section 5 presents a brief conclusion; and Appendix A contains a certain auxiliary result.

\section{$2 \quad$ Setting}

This section details the form of the SDG for which our SMP will be constructed. For the sake of simplicity we consider the problem in its reduced one-dimensional form: that is we consider the driving Brownian motion, the state process and its coefficient functions $\left\{b, \sigma, \phi_{b}, \phi_{\sigma}\right\}$, and all the functions in each agent's performance functional $\left\{f_{i}, g_{i}, \phi_{f_{i}}, \phi_{g_{i}} \mid i \in\{1, \ldots, N\}\right\}$ as being valued in $\mathbb{R}$. A version of this paper which addresses the more general multidimensional form of the problem is [10].

Fix any $T \in(0, \infty)$ and $x_{0} \in \mathbb{R}$. For each $i \in\{1, \ldots, N\}$ let $\left(U_{i}, d_{i}\right)$ be a complete separable metric space, and denote $U:=\prod_{i=1}^{N} U_{i}$. Let $(\Omega, \mathcal{F}, \mathbb{P})$ be a complete probability space, and $B: \Omega \times[0, T] \rightarrow \mathbb{R}$ a standard Brownian motion with respect to its augmented natural filtration $\mathbb{F}:=\left\{\mathcal{F}_{t} \mid t \in[0, T]\right\}$ (that is known to be right-continuous), and which $\mathbb{P}$-almost surely is taken as having continuous paths. It is assumed that $\mathcal{F}_{T}=\mathcal{F}$. Given any filtration $\mathbb{O}=\left\{\mathcal{O}_{t} \mid t \in[0, T]\right\}$ for $(\Omega, \mathcal{F}, \mathbb{P})$, then we denote by $\mathcal{P}(\mathbb{O})$ the corresponding predictable $\sigma$-algebra on $\Omega \times[0, T]$ with respect to $\mathbb{O}$.

We assume throughout that the collection of coefficient functions has the properties detailed in Assumption 2.1 below.

Assumption 2.1. The functions

$$
\left\{\begin{array}{l}
\left\{b, \sigma, f_{i}: \Omega \times[0, T] \times \mathbb{R}^{2} \times U \rightarrow \mathbb{R} \mid i \in\{1, \ldots, N\}\right\} \\
\left\{\phi_{b}, \phi_{\sigma}, \phi_{f_{i}}: \Omega \times[0, T] \times \mathbb{R} \times U \rightarrow \mathbb{R} \mid i \in\{1, \ldots, N\}\right\} \\
\left\{g_{i}: \Omega \times \mathbb{R}^{2} \rightarrow \mathbb{R} \mid i \in\{1, \ldots, N\}\right\} \\
\left\{\phi_{g_{i}}: \Omega \times \mathbb{R} \rightarrow \mathbb{R} \mid i \in\{1, \ldots, N\}\right\}
\end{array}\right.
$$


have the following properties:

(i)

$$
\begin{array}{rll}
b, \sigma,\left\{f_{i} \mid i \in\{1, \ldots, N\}\right\} & \text { are } & \mathcal{P}(\mathbb{F}) \times \mathcal{B}\left(\mathbb{R}^{2} \times U\right) \mid \mathcal{B}(\mathbb{R}) \text {-measurable } ; \\
\phi_{b}, \phi_{\sigma},\left\{\phi_{f_{i}} \mid i \in\{1, \ldots, N\}\right\} & \text { are } & \mathcal{P}(\mathbb{F}) \times \mathcal{B}(\mathbb{R} \times U) \mid \mathcal{B}(\mathbb{R}) \text {-measurable } \\
\left\{g_{i} \mid i \in\{1, \ldots, N\}\right\} & \text { are } & \mathcal{F} \times \mathcal{B}\left(\mathbb{R}^{2}\right) \mid \mathcal{B}(\mathbb{R}) \text {-measurable; } \\
\left\{\phi_{g_{i}} \mid i \in\{1, \ldots, N\}\right\} & \text { are } & \mathcal{F} \times \mathcal{B}(\mathbb{R}) \mid \mathcal{B}(\mathbb{R}) \text {-measurable. }
\end{array}
$$

(ii) For $\mathbb{P}$-almost all $\omega \in \Omega$, the functions:

$$
\begin{array}{rll}
(x, y) \mapsto b(\omega, t, x, y, u), \sigma(\omega, t, x, y, u), f_{i}(\omega, t, x, y, u) & \text { are in } & \mathcal{C}^{2}\left(\mathbb{R}^{2} ; \mathbb{R}\right), \\
x \mapsto \phi_{b}(\omega, t, x, u), \phi_{\sigma}(\omega, t, x, u), \phi_{f_{i}}(\omega, t, x, u) & \text { are in } & \mathcal{C}^{2}(\mathbb{R} ; \mathbb{R}), \\
(x, y) \mapsto g_{i}(\omega, x, y) & \text { are in } & \mathcal{C}^{2}\left(\mathbb{R}^{2} ; \mathbb{R}\right), \\
x \mapsto \phi_{g_{i}}(\omega, x) & \text { are in } & \mathcal{C}^{2}(\mathbb{R} ; \mathbb{R}),
\end{array}
$$

uniformly for any $t \in[0, T]$ and $u \in U$, for all $i \in\{1, \ldots, N\}$.

(iii) There exists a constant $c>0$ such that:

$$
\begin{gathered}
\max \left\{|\psi(\omega, t, 0,0, u)|,\left|\partial_{x} \psi(\omega, t, x, y, u)\right|,\left|\partial_{y} \psi(\omega, t, x, y, u)\right|,\left|\partial_{x, x}^{(2)} \psi(\omega, t, x, y, u)\right|,\right. \\
\left|\partial_{x, y}^{(2)} \psi(\omega, t, x, y, u)\right|,\left|\partial_{y, y}^{(2)} \psi(\omega, t, x, y, u)\right|,\left|\phi_{\psi}(\omega, t, 0, u)\right|, \\
\left|\partial \phi_{\psi}(\omega, t, x, u)\right|,\left|\partial^{(2)} \phi_{\psi}(\omega, t, x, u)\right| \psi \in\left\{b, \sigma, f_{i} \mid i \in\{1, \ldots, N\}\right\} \leq c \\
\max \left\{\left|\partial_{x, x}^{(2)} \psi(\omega, t, x, y, u)-\partial_{x, x}^{(2)} \psi\left(\omega, t, x^{\prime}, y^{\prime}, u\right)\right|,\left|\partial_{x, y}^{(2)} \psi(\omega, t, x, y, u)-\partial_{x, y}^{(2)} \psi\left(\omega, t, x^{\prime}, y^{\prime}, u\right)\right|,\right. \\
\left|\partial_{y, y}^{(2)} \psi(\omega, t, x, y, u)-\partial_{y, y}^{(2)} \psi\left(\omega, t, x^{\prime}, y^{\prime}, u\right)\right|,\left|\partial^{(2)} \phi_{\psi}(\omega, t, x, u)-\partial^{(2)} \phi_{\psi}\left(\omega, t, x^{\prime}, u\right)\right| \\
\mid \psi \in\left\{b, \sigma, f_{i} \mid i \in\{1, \ldots, N\}\right\} \leq c\left(\left|x-x^{\prime}\right|+\left|y-y^{\prime}\right|\right) \\
\max \left\{\left|\partial_{x} g_{i}(\omega, x, y)\right|,\left|\partial_{y} g_{i}(\omega, x, y)\right|,\left|\partial_{x, x}^{(2)} g_{i}(\omega, x, y)\right|,\left|\partial_{x, y}^{(2)} g_{i}(\omega, x, y)\right|,\right. \\
\left.\left|\partial_{y, y}^{(2)} g_{i}(\omega, x, y)\right|,\left|\partial \phi_{g_{i}}(\omega, x)\right|,\left|\partial^{(2)} \phi_{g_{i}}(\omega, x)\right| \mid i \in\{1, \ldots, N\}\right\} \leq c \\
\max \left\{\left|\partial_{x, x}^{(2)} g_{i}(\omega, x, y)-\partial_{x, x}^{(2)} g_{i}\left(\omega, x^{\prime}, y^{\prime}\right)\right|,\left|\partial_{x, y}^{(2)} g_{i}(\omega, x, y)-\partial_{x, y}^{(2)} g_{i}\left(\omega, x^{\prime}, y^{\prime}\right)\right|,\right. \\
\left|\partial_{y, y}^{(2)} g_{i}(\omega, x, y)-\partial_{y, y}^{(2)} g_{i}\left(\omega, x^{\prime}, y^{\prime}\right)\right|,\left|\partial^{(2)} \phi_{g_{i}}(\omega, x)-\partial^{(2)} \phi_{g_{i}}\left(\omega, x^{\prime}\right)\right| \\
\mid i \in\{1, \ldots, N\}\} \leq c\left(\left|x-x^{\prime}\right|+\left|y-y^{\prime}\right|\right)
\end{gathered}
$$

for $\mathbb{P}$-almost all $\omega \in \Omega$ uniformly for all $t \in[0, T], x, x^{\prime}, y, y^{\prime} \in \mathbb{R}$ and $u \in U$.

(iv) There exists a subset $\Omega_{0} \subseteq \Omega$ with $\mathbb{P}\left(\Omega_{0}\right)=1$ such that for each $\psi \in\left\{b, \sigma, f_{i} \mid i \in\{1, \ldots, N\}\right\}$ each map $u \mapsto \psi(\omega, t, x, y, u)$ and $u \mapsto \phi_{\psi}(\omega, t, x, u)$ is continuous and bounded uniformly for all $(\omega, t) \in \Omega_{0} \times[0, T]$ and $x, y \in \mathbb{R}$.

Definition 2.2. Let $\left\{\mathbb{G}_{i} \mid i \in\{1, \ldots, N\}\right\}$ be a collection of-not necessarily equal-filtrations, where for each $i \in\{1, \ldots, N\}$ the $\mathbb{G}_{i}:=\left\{\mathcal{G}_{i, t} \mid t \in[0, T]\right\}$ is complete, right-continuous, and a subfiltration of $\mathbb{F}$. For each $i \in\{1, \ldots, N\}$, we define $\mathcal{A}_{i}$, the class of admissible controls for the $i^{\text {th }}$-agent, as the set of all $\mathcal{P}\left(\mathbb{G}_{i}\right) \mid \mathcal{B}\left(U_{i}\right)$-measurable processes $u_{i}: \Omega \times[0, T] \rightarrow U_{i}$.

Proposition 2.3 (cf. Theorem 4.1 in [7]). Under Assumption 2.1, for each $u:=\left(u_{1}, \ldots, u_{N}\right) \in$ $\prod_{i=1}^{N} \mathcal{A}_{i}$ there exists a unique - up to indistinguishability—stochastic process $X^{(u)}: \Omega \times[0, T] \rightarrow \mathbb{R}$ that $\mathbb{P}$-almost surely has continuous paths and which:

- is a strong $\mathbb{F}$-adapted solution to the following mean-field type SDE

$$
\begin{aligned}
X^{(u)}(t)=x_{0}+ & \int_{0}^{t} b\left(s, X^{(u)}(s), \int_{\Omega} \phi_{b}\left(\omega^{\prime}, s, X^{(u)}\left(\omega^{\prime}, s\right), u\left(\omega^{\prime}, s\right)\right) \mathbb{P}\left(\mathrm{d} \omega^{\prime}\right), u(s)\right) \mathrm{d} s \\
& +\int_{0}^{t} \sigma\left(s, X^{(u)}(s), \int_{\Omega} \phi_{\sigma}\left(\omega^{\prime}, s, X^{(u)}\left(\omega^{\prime}, s\right), u\left(\omega^{\prime}, s\right)\right) \mathbb{P}\left(\mathrm{d} \omega^{\prime}\right), u(s)\right) \mathrm{d} B(s)
\end{aligned}
$$

for $\mathbb{P}$-almost all $\omega \in \Omega$ uniformly for all $t \in[0, T]$; and 
- is such that for each $p \in[1, \infty)$ then $\mathbb{E}_{\mathbb{P}}\left[\sup _{t \in[0, T]}\left|X^{(u)}(t)\right|^{p}\right]<\infty$.

Proof. The result can be established by making suitable slight adaptations to the standard techniques for solutions to (Brownian motion driven) Lipschitz SDEs, in particular using a Picard-iteration method for the existence part of the result.

In this paper we focus solely on the form of $N$-agent SDG that is specified by the state dynamics defined in the mean-field SDE (1) and by the performance functionals given in Definition 2.4 below.

Definition 2.4. Under Assumption 2.1, for each $i \in\{1, \ldots, N\}$ define the performance functional $J_{i}: \prod_{\ell=1}^{N} \mathcal{A}_{\ell} \rightarrow \mathbb{R}$, for the $i^{\text {th }}$-agent, by

$$
\begin{aligned}
J_{i}(u):=\mathbb{E}_{\mathbb{P}}\left[\int_{0}^{T} f_{i}\left(t, X^{(u)}(t), \int_{\Omega} \phi_{f_{i}}\left(\omega^{\prime}, t, X^{(u)}\left(\omega^{\prime}, t\right), u\left(\omega^{\prime}, t\right)\right) \mathbb{P}\left(\mathrm{d} \omega^{\prime}\right), u(t)\right) \mathrm{d} t\right. & \\
& \left.+g_{i}\left(X^{(u)}(T), \int_{\Omega} \phi_{g_{i}}\left(\omega^{\prime}, X^{(u)}\left(\omega^{\prime}, T\right)\right) \mathbb{P}\left(\mathrm{d} \omega^{\prime}\right)\right)\right]
\end{aligned}
$$

for all $u \in \prod_{\ell=1}^{N} \mathcal{A}_{\ell}$, where for each $u \in \prod_{\ell=1}^{N} \mathcal{A}_{\ell}$ the controlled state process $X^{(u)}: \Omega \times[0, T] \rightarrow \mathbb{R}$ is defined as the solution to the corresponding mean-field type SDE (1) in the sense of Proposition 2.3.

\section{A Stochastic Maximum Principle}

Recall that a Nash equilibrium, for the SDG detailed by equations (1) and (2), is an $N$-tuple of admissible controls $u^{*}:=\left(u_{1}^{*}, \ldots, u_{N}^{*}\right) \in \prod_{\ell=1}^{N} \mathcal{A}_{\ell}$ such that for each $i \in\{1, \ldots, N\}$ then

$$
J_{i}\left(u^{*}\right) \geq J_{i}\left(u_{i}^{*}\left(u_{i}\right)\right)
$$

for all $u_{i} \in \mathcal{A}_{i}$, where $u_{i}^{*}\left(u_{i}\right):=\left(u_{i}^{*}\left(u_{i}\right)_{1}, \ldots, u_{i}^{*}\left(u_{i}\right)_{N}\right) \in \prod_{\ell=1}^{N} \mathcal{A}_{\ell}$ is defined by

$$
u_{i}^{*}\left(u_{i}\right)_{j}:= \begin{cases}u_{i} & \text { if } j=i \\ u_{j}^{*} & \text { for all } j \in\{1, \ldots, N\} \backslash\{i\} .\end{cases}
$$

Assumption 3.1. Under Assumption 2.1, assume that there exists a $u^{*}=\left(u_{1}^{*}, \ldots, u_{N}^{*}\right) \in \prod_{\ell=1}^{N} \mathcal{A}_{\ell}$ which is a Nash equilibrium for the SDG of equations (1) and (2). Let $X^{*}: \Omega \times[0, T] \rightarrow \mathbb{R}$ be the state process corresponding to the $N$-tuple of admissible controls $u^{*}, X^{*}:=X^{\left(u^{*}\right)}$.

We make use of the following notation.

Notation 3.2. Suppose Assumptions 2.1 and 3.1 hold. For each function $\psi \in\left\{b, \sigma, f_{i} \mid i \in\{1, \ldots, N\}\right\}$ define $\partial \psi: \Omega \times[0, T] \times \mathbb{R}^{2} \times U \times \mathbb{R}^{2} \rightarrow \mathbb{R}$ and $\partial^{(2)} \psi: \Omega \times[0, T] \times \mathbb{R}^{2} \times U \times \mathbb{R}^{3} \rightarrow \mathbb{R}$ by

$$
\begin{aligned}
\partial \psi(\omega, t, x, y, u, v, w) & :=\partial_{x} \psi(\omega, t, x, y, u) v+\partial_{y} \psi(\omega, t, x, y, u) w \\
\partial^{(2)} \psi(\omega, t, x, y, u, v, w, z):=\partial_{x, x}^{(2)} \psi & (\omega, t, x, y, u) v^{2}+2 \partial_{x, y}^{(2)} \psi(\omega, t, x, y, u) v w \\
& +\partial_{y, y}^{(2)} \psi(\omega, t, x, y, u) w^{2}+\partial_{y} \psi(\omega, t, x, y, u) z
\end{aligned}
$$

for $\mathbb{P}$-almost all $\omega \in \Omega$ uniformly for all $t \in[0, T], x, y, v, w, z \in \mathbb{R}$, and $u \in U$. Define, for each $i \in\{1, \ldots, N\}$, the functions $\partial g_{i}: \Omega \times \mathbb{R}^{4} \rightarrow \mathbb{R}$ and $\partial^{(2)} g_{i}: \Omega \times \mathbb{R}^{5} \rightarrow \mathbb{R}$ by

$$
\begin{aligned}
\partial g_{i}(\omega, x, y, v, w) & :=\partial_{x} g_{i}(\omega, x, y) v+\partial_{y} g_{i}(\omega, x, y) w \\
\partial^{(2)} g_{i}(\omega, x, y, v, w, z) & :=\partial_{x, x}^{(2)} g_{i}(\omega, x, y) v^{2}+2 \partial_{x, y}^{(2)} g_{i}(\omega, x, y) v w+\partial_{y, y}^{(2)} g_{i}(\omega, x, y) w^{2}+\partial_{y} g_{i}(\omega, x, y) z
\end{aligned}
$$

for $\mathbb{P}$-almost all $\omega \in \Omega$ uniformly for all $t \in[0, T], x, y, v, w, z \in \mathbb{R}$, and $u \in U$.

Define the shortened notation:

$$
\begin{gathered}
\psi(\omega, t):=\psi\left(\omega, t, X^{*}(\omega, t), \int_{\Omega} \phi_{\psi}\left(\omega^{\prime}, t, X^{*}\left(\omega^{\prime}, t\right), u^{*}\left(\omega^{\prime}, t\right)\right) \mathbb{P}\left(\mathrm{d} \omega^{\prime}\right), u^{*}(\omega, t)\right) \\
\text { for all } \psi \in\left\{\lambda, \partial_{x} \lambda, \partial_{y} \lambda, \partial_{x, x}^{(2)} \lambda, \partial_{x, y}^{(2)} \lambda, \partial_{y, y}^{(2)} \lambda \mid \lambda=b, \sigma, f_{1}, \ldots, f_{N}\right\} \\
\psi(\omega, t):=\psi\left(\omega, t, X^{*}(\omega, t), u^{*}(\omega, t)\right)
\end{gathered}
$$

for all $\psi \in\left\{\lambda, \partial \lambda, \partial^{(2)} \lambda \mid \lambda=\phi_{b}, \phi_{\sigma}, \phi_{f_{1}}, \ldots, \phi_{f_{N}}\right\}$; 
$\bullet$

$$
\psi(\omega):=\psi\left(\omega, X^{*}(\omega, T), \int_{\Omega} \phi_{\psi}\left(\omega^{\prime}, X^{*}\left(\omega^{\prime}, T\right)\right) \mathbb{P}\left(\mathrm{d} \omega^{\prime}\right)\right)
$$

for all $\psi \in\left\{g_{i}, \partial_{x} g_{i}, \partial_{y} g_{i}, \partial_{x, x}^{(2)} g_{i}, \partial_{x, y}^{(2)} g_{i}, \partial_{y, y}^{(2)} g_{i} \mid i \in\{1, \ldots, N\}\right\}$;

$$
\psi(\omega):=\psi\left(\omega, X^{*}(\omega, T)\right)
$$

for all $\psi \in\left\{\phi_{g_{i}}, \partial \phi_{g_{i}}, \partial^{(2)} \phi_{g_{i}} \mid i \in\{1, \ldots, N\}\right\}$.

Note that the definitions of the spaces $\mathcal{S}^{2}(\Omega \times[0, T] ; \mathbb{R})$ and $\mathcal{H}^{2}(\Omega \times[0, T] ; \mathbb{R})$ are given in Definition 4.1 .

Definition 3.3. Suppose Assumptions 2.1 and 3.1 hold. For each $i \in\{1, \ldots, N\}$ define the map $h_{i}: \Omega \times \Omega \times[0, T] \times \mathbb{R}^{4} \rightarrow \mathbb{R}$ by

$$
\begin{aligned}
h_{i}\left(\omega, \omega^{\prime}, t, v, v^{\prime}, w, w^{\prime}\right):=\partial_{x} f_{i}(\omega, t)+\partial_{y} f_{i}\left(\omega^{\prime}, t\right) \partial \phi_{f_{i}}(\omega, t) & +v \partial_{x} b(\omega, t)+v^{\prime} \partial_{y} b\left(\omega^{\prime}, t\right) \partial \phi_{b}(\omega, t) \\
& +w \partial_{x} \sigma(\omega, t)+w^{\prime} \partial_{y} \sigma\left(\omega^{\prime}, t\right) \partial \phi_{\sigma}(\omega, t)
\end{aligned}
$$

for all $\left(\omega, \omega^{\prime}\right) \in \Omega_{0} \times \Omega_{0}, t \in[0, T]$, and $v, v^{\prime}, w, w^{\prime} \in \mathbb{R}$, for some $\Omega_{0} \subseteq \Omega$ such that $\mathbb{P}\left(\Omega_{0}\right)=1$. Then for each $i \in\{1, \ldots, N\}$ define the processes $\left(p_{i}, q_{i}\right) \in \mathcal{S}^{2}(\Omega \times[0, T] ; \mathbb{R}) \times \mathcal{H}^{2}(\Omega \times[0, T] ; \mathbb{R})$ as the solution of the following MFBSDE

$$
\left\{\begin{aligned}
p_{i}(\omega, t)= & p_{i}(\omega, T)+\int_{t}^{T} \int_{\Omega} h_{i}\left(\left(\omega, \omega^{\prime}\right), s, p_{i}(\omega, s), p_{i}\left(\omega^{\prime}, s\right), q_{i}(\omega, s), q_{i}\left(\omega^{\prime}, s\right)\right) \mathbb{P}\left(\mathrm{d} \omega^{\prime}\right) \mathrm{d} s \\
& -\int_{t}^{T} q_{i}(\omega, s) \mathrm{d} B(\omega, s) \\
p_{i}(\omega, T)= & \partial_{x} g_{i}(\omega)+\mathbb{E}_{\mathbb{P}}\left[\partial_{y} g_{i}\right] \partial \phi_{g_{i}}(\omega)
\end{aligned}\right.
$$

for $\mathbb{P}$-almost all $\omega \in \Omega$ uniformly for all $t \in[0, T]$.

For each $i \in\{1, \ldots, N\}$ define the map $l_{i}: \Omega \times[0, T] \times \mathbb{R}^{2} \rightarrow \mathbb{R}$ by

$$
\begin{aligned}
l_{i}(t, v, w):=\partial_{x, x}^{(2)} f_{i}(t)+\mathbb{E}_{\mathbb{P}}\left[\partial_{y} f_{i}(t)\right] \partial^{(2)} \phi_{f_{i}}(t)+p_{i}(t) \partial_{x, x}^{(2)} b(t)+\mathbb{E}_{\mathbb{P}}\left[p_{i}(t) \partial_{y} b(t)\right] \partial^{(2)} \phi_{b}(t) \\
+q_{i}(t) \partial_{x, x}^{(2)} \sigma(t)+\mathbb{E}_{\mathbb{P}}\left[q_{i}(t) \partial_{y} \sigma(t)\right] \partial^{(2)} \phi_{\sigma}(t)+v\left(2 \partial_{x} b(t)+\left(\partial_{x} \sigma(t)\right)^{2}\right)+2 w \partial_{x} \sigma(t)
\end{aligned}
$$

for $\mathbb{P}$-almost all $\omega \in \Omega$ uniformly for all $t \in[0, T]$, and $v, w \in \mathbb{R}$. Then for each $i \in\{1, \ldots, N\}$ define the processes $\left(P_{i}, Q_{i}\right) \in \mathcal{S}^{2}(\Omega \times[0, T] ; \mathbb{R}) \times \mathcal{H}^{2}(\Omega \times[0, T] ; \mathbb{R})$ as the solution of the following BSDE

$$
\left\{\begin{aligned}
P_{i}(t) & =P_{i}(T)+\int_{t}^{T} l_{i}\left(s, P_{i}(s), Q_{i}(s)\right) \mathrm{d} s-\int_{t}^{T} Q_{i}(s) \mathrm{d} B(s) \\
P_{i}(T) & =\partial_{x, x}^{(2)} g_{i}+\mathbb{E}_{\mathbb{P}}\left[\partial_{y} g_{i}\right] \partial^{(2)} \phi_{g_{i}}
\end{aligned}\right.
$$

for $\mathbb{P}$-almost all $\omega \in \Omega$ uniformly for all $t \in[0, T]$.

Remark 3.4. Note that given the assumed properties in Assumption 2.1 then:

- For each $i \in\{1, \ldots, N\}$, the processes $\left(p_{i}, q_{i}\right)$ in Definition 3.3 are well-defined, this can be shown by making a minor modification to Theorem 3.1 in [7].

- For each $i \in\{1, \ldots, N\}$, the processes $\left(P_{i}, Q_{i}\right)$ in Definition 3.3 are well-defined due to known results in the theory of BSDEs, see, for example, subsection 2.1 of [8].

Assumption 3.5. Suppose Assumptions 2.1 and 3.1 hold. Assume that, for each $i \in\{1, \ldots, N\}$, there exist representations of the equivalence classes $q_{i}, Q_{i} \in \mathcal{H}^{2}(\Omega \times[0, T] ; \mathbb{R})$ which are defined $\mathbb{P}$ almost surely uniformly for all $t \in[0, T]$. In the following we take each $q_{i}$ and $Q_{i}$ as denoting those representations.

Also, suppose that for each $i \in\{1, \ldots, N\}$ there exists a $\mathcal{P}\left(\mathbb{G}_{i}\right) \times \mathcal{B}\left(U_{i}\right) \mid \mathcal{B}(\mathbb{R})$-measurable process $H_{i}: \Omega \times[0, T] \times U_{i} \rightarrow \mathbb{R}$ such that:

- the map $H_{i}(\omega, t, \cdot): U_{i} \rightarrow \mathbb{R}$ is continuous and bounded, uniformly for all $(\omega, t) \in \Omega \times[0, T]$;

- for any given $u_{i} \in \mathcal{A}_{i}$ then

$$
\begin{aligned}
H_{i}\left(\omega, t, u_{i}(\omega, t)\right)=\rho_{\mathbb{G}_{i}} & {\left[f_{i}\left(\cdot, X^{*}(\cdot), \int_{\Omega} \phi_{f_{i}}\left(\omega^{\prime}, \cdot, X^{*}\left(\omega^{\prime}, \cdot\right), u_{i}^{*}\left(u_{i}\right)\left(\omega^{\prime}, \cdot\right)\right) \mathbb{P}\left(\mathrm{d} \omega^{\prime}\right), u_{i}^{*}\left(u_{i}\right)(\cdot)\right)\right.} \\
+ & p_{i}(\cdot) b\left(\cdot, X^{*}(\cdot), \int_{\Omega} \phi_{b}\left(\omega^{\prime}, \cdot, X^{*}\left(\omega^{\prime}, \cdot\right), u_{i}^{*}\left(u_{i}\right)\left(\omega^{\prime}, \cdot\right)\right) \mathbb{P}\left(\mathrm{d} \omega^{\prime}\right), u_{i}^{*}\left(u_{i}\right)(\cdot)\right) \\
+ & q_{i}(\cdot) \sigma\left(\cdot, X^{*}(\cdot), \int_{\Omega} \phi_{\sigma}\left(\omega^{\prime}, \cdot, X^{*}\left(\omega^{\prime}, \cdot\right), u_{i}^{*}\left(u_{i}\right)\left(\omega^{\prime}, \cdot\right)\right) \mathbb{P}\left(\mathrm{d} \omega^{\prime}\right), u_{i}^{*}\left(u_{i}\right)(\cdot)\right) \\
+ & P_{i}(\cdot)\left(\sigma\left(\cdot, X^{*}(\cdot), \int_{\Omega} \phi_{\sigma}\left(\omega^{\prime}, \cdot, X^{*}\left(\omega^{\prime}, \cdot\right), u_{i}^{*}\left(u_{i}\right)\left(\omega^{\prime}, \cdot\right)\right) \mathbb{P}\left(\mathrm{d} \omega^{\prime}\right), u_{i}^{*}\left(u_{i}\right)(\cdot)\right)\right. \\
& \left.\left.-\sigma\left(\cdot, X^{*}(\cdot), \int_{\Omega} \phi_{\sigma}\left(\omega^{\prime}, \cdot, X^{*}\left(\omega^{\prime}, \cdot\right), u^{*}\left(\omega^{\prime}, \cdot\right)\right) \mathbb{P}\left(\mathrm{d} \omega^{\prime}\right), u^{*}(\cdot)\right)\right)^{2}\right](\omega, t)
\end{aligned}
$$


for $\mathbb{P}$-almost all $\omega \in \Omega$ uniformly for all $t \in[0, T]$, where $\rho_{\mathbb{G}_{i}}[\cdot]$ denotes the $\mathcal{P}\left(\mathbb{G}_{i}\right)$-predictable projection.

Theorem 3.6 presents the SMP which provides necessary conditions for the existence of Nash equilibria in the considered SDG.

Theorem 3.6. Under Assumptions 2.1, 3.1 and 3.5, for $u^{*} \in \prod_{\ell=1}^{N} \mathcal{A}_{\ell}$ to be a Nash equilibrium for the SDG of equations (1) and (2), as assumed, then it is necessary that for each $i \in\{1, \ldots, N\}$ the following SMP is satisfied

$$
\max _{v \in U_{i}} H_{i}(\omega, t, v)=H_{i}\left(\omega, t, u_{i}^{*}(\omega, t)\right)
$$

for $\mathbb{P} \otimes$ Leb-almost all $(\omega, t) \in \Omega \times[0, T]$.

Proof. Fix any arbitrary $i \in\{1, \ldots, N\}$ and any arbitrary admissible control $u_{i} \in \mathcal{A}_{i}$ for the $i^{\text {th }}$-agent. Let $u_{i}^{*}\left(u_{i}^{(r, \epsilon)}\right)=\left(u_{i}^{*}\left(u_{i}^{(r, \epsilon)}\right)_{1}, \ldots, u_{i}^{*}\left(u_{i}^{(r, \epsilon)}\right)_{N}\right) \in \prod_{\ell=1}^{N} \mathcal{A}_{\ell}$ denote

$$
u_{i}^{*}\left(u_{i}^{(r, \epsilon)}\right)_{j}:= \begin{cases}u_{i}^{(r, \epsilon)} & , \text { if } j=i \\ u_{j}^{*} & , \text { if } j \in\{1, \ldots, N\} \backslash\{i\}\end{cases}
$$

where the family of spike-variations, $\left\{u_{i}^{(r, \epsilon)} \mid r \in[0, T), \epsilon \in(0, T-r]\right\}$, of the $i^{t h}$-agent's control $u_{i}^{*}$, is defined by

$$
u_{i}^{(r, \epsilon)}(t):=u_{i}^{*}(t)\left(1-\mathbf{1}_{(r, r+\epsilon]}(t)\right)+u_{i}(t) \mathbf{1}_{(r, r+\epsilon]}(t)
$$

for all $(\omega, t) \in \Omega \times[0, T]$. We aim to show that

$$
\lim _{\epsilon \downarrow 0} \epsilon^{-1}\left(J_{i}\left(u_{i}^{*}\left(u_{i}^{(r, \epsilon)}\right)\right)-J_{i}\left(u^{*}\right)\right)=\mathbb{E}_{\mathbb{P}}\left[H_{i}\left(r, u_{i}(r)\right)-H_{i}\left(r, u_{i}^{*}(r)\right)\right]
$$

for Leb-almost all $r \in[0, T]$, since if this were true then under the assumption that the $N$-tuple $u^{*}$ of admissible controls is a Nash equilibrium, it would follow that for each $u_{i} \in \mathcal{A}_{i}$ then

$$
\mathbb{E}_{\mathbb{P}}\left[H_{i}\left(r, u_{i}(r)\right)-H_{i}\left(r, u_{i}^{*}(r)\right)\right] \leq 0
$$

for Leb-almost all $r \in[0, T]$. This follows the general principle of the Peng [21] method when transfered to the SDG setting, and is analogous to the method used in [19] and that used in [5].

The main part of this proof is concerned with the calculation of the limit in equation (7). The approach we take to do this is also developed on the general idea of that used in [21] for the corresponding problem. That approach being to expand the difference between the relevant two performance functionals into terms of certain types, and to introduce certain adjoint processes represented by backward stochastic equations (certain BSDEs in the case of [21]), to help one calculate the limit as required. The approach we use is also related in certain respects to that in [19] and that in [5], which are themselves related, again in certain respects, to the original work of Peng [21].

Let $\left\{X^{(r, \epsilon)}: \Omega \times[0, T] \rightarrow \mathbb{R} \mid r \in[0, T), \epsilon \in(0, T-r]\right\}$ denote the family of controlled state processes corresponding to the controls $u_{i}^{*}\left(u_{i}^{(r, \epsilon)}\right)$. Also, let $\left\{\zeta^{(r, \epsilon)}: \Omega \times[0, T] \rightarrow \mathbb{R} \mid r \in[0, T), \epsilon \in(0, T-r]\right\}$ be the family of processes defined, for each $r \in[0, T)$ and $\epsilon \in(0, T-r]$, by $\zeta^{(r, \epsilon)}:=X^{(r, \epsilon)}-X^{*}$.

We use an expansion of the process $\zeta^{(r, \epsilon)}$ (see Lemma A.1), which, in regards to its format, is more akin to that in [20] than that which is used in [21] and [5], but which we use in a similar role. Given Lemma A.1 and Assumption 3.1, we then decompose the term $J_{i}\left(u_{i}^{*}\left(u_{i}^{(r, \epsilon)}\right)\right)-J_{i}\left(u^{*}\right)$ into a sum of four components:

- a linear-type term with respect to the variables $\zeta^{(r, \epsilon)}$;

- a quadratic-type term with respect to the variables $\zeta^{(r, \epsilon)}$;

- a type of higher order term which we will show is of the order $o(\epsilon)$ as $\epsilon \downarrow 0$; and

- a term for which the required limit may be calculated in a relatively simple manner;

An analogous form of decomposition may also be identified in [21] and [5]. The decomposition used in [19], that is in the sense of Lemma 3.1 of [19], is, to some extent, of different type of format.

Our linear-type term takes the form

$$
\mathbb{E}_{\mathbb{P}}\left[\int_{r+\epsilon}^{T} Y_{1}(s) \zeta^{(r, \epsilon)}(s) \mathrm{d} s+Y_{1}(T) \zeta^{(r, \epsilon)}(T)\right]
$$

for certain factors $Y_{1}$. The procedure to deal with it is similar to that in [5] and is analogous to that which was first developed in [21], for their corresponding type terms. In [21], it was noted that 
the relevant linear-type component is in fact a linear functional of another term, and that when this linear functional is written (by the Riesz representation theorem) as an inner-product between that other term and its relevant adjoint term, then one may calculate, as desired, the limit as $\epsilon \downarrow 0$ of the linear-type term multiplied by $\epsilon^{-1}$. With the expansion of $\zeta^{(r, \epsilon)}$ that we use in this paper, we do not fully calculate the corresponding limit at this stage, but use the procedure to rewrite the linear-type term as a sum of elements that belong to the three other categories in the decomposition listed above. In [21], it is also described how this relevant adjoint term may be given by the solution of a certain linear BSDE. In this paper we introduce all our adjoint processes directly as solutions to the relevant backward stochastic equations. In our case, and as it is in [5], the form of the required backward stochastic equation for this linear-type term is that of a mean-field backward stochastic differential equation - a form of equation that has been studied in [6] and [7].

In [21], the relevant quadratic-type term is also treated using an adjoint representation technique, and it is shown that the adjoint term can be given by the solution of another linear BSDE. We apply a comparable form of procedure in order to deal with the quadratic-type term in our problem. Although, the procedure is, to some extent, complicated by the mean-field nature of our problem. Here we have two forms of quadratic-type terms: those of a form

$$
\mathbb{E}_{\mathbb{P}}\left[\int_{r+\epsilon}^{T} Y_{2,1}(s) \zeta^{(r, \epsilon)}(s)^{2} \mathrm{~d} s+Y_{2,1}(T) \zeta^{(r, \epsilon)}(T)^{2}\right]
$$

for certain factors $Y_{2,1}$; and those of a form

$$
\int_{r+\epsilon}^{T} \mathbb{E}_{\mathbb{P}}\left[Y_{2,2,1}(s) \zeta^{(r, \epsilon)}(s)\right] \mathbb{E}_{\mathbb{P}}\left[Y_{2,2,2}(s) \zeta^{(r, \epsilon)}(s)\right] \mathrm{d} s+\mathbb{E}_{\mathbb{P}}\left[Y_{2,2,1}(T) \zeta^{(r, \epsilon)}(T)\right] \mathbb{E}_{\mathbb{P}}\left[Y_{2,2,2}(T) \zeta^{(r, \epsilon)}(T)\right]
$$

for certain factors $Y_{2,2,1}$ and $Y_{2,2,2}$. A comparable situation exists in [5] also.

We treat the first form of quadratic-type term using an adjoint process that is defined as the solution of a certain BSDE, which, again, is similar to how the relevant first form of quadratic-type term is treated in [5], and related to how the relevant quadratic-type term is treated in [21]. We treat the second form of quadratic-type term by applying an adjoint representation procedure on the completion of a product probability space formed from $(\Omega, \mathcal{F}, \mathbb{P})$ and a copy of itself. The relevant adjoint process is given by the solution of an equation that is of a class of backward stochastic equations that we introduce in this paper (see Section 4), and which we term conditional mean-field backward stochastic differential equations (CMFBSDEs). By using this third form of adjoint process we are able to show that the limit as $\epsilon \downarrow 0$ of this second form of quadratic-type term multiplied by $\epsilon^{-1}$, will be null. Thus these third-type adjoint processes do not appear in our final SMP equations. In [5] the authors establish bounds on their relevant second form of quadratic-type term, to imply that it is of order $o(\epsilon)$.

Define the shortened notation (using the notation $u_{i}^{*}\left(u_{i}\right)$ as it is defined in equation (3)):

$$
\begin{gathered}
\partial \psi(\omega, t):=\partial \psi\left(\omega, t, X^{*}(\omega, t), \int_{\Omega} \phi_{\psi}\left(\omega^{\prime}, t, X^{*}\left(\omega^{\prime}, t\right), u^{*}\left(\omega^{\prime}, t\right)\right) \mathbb{P}\left(\mathrm{d} \omega^{\prime}\right), u^{*}(\omega, t),\right. \\
\left.\zeta^{(r, \epsilon)}(\omega, t), \int_{\Omega} \partial \phi_{\psi}\left(\omega^{\prime}, t, X^{*}\left(\omega^{\prime}, t\right), u^{*}\left(\omega^{\prime}, t\right)\right) \zeta^{(r, \epsilon)}\left(\omega^{\prime}, t\right) \mathbb{P}\left(\mathrm{d} \omega^{\prime}\right)\right) \\
\partial^{(2)} \psi(\omega, t):=\partial^{(2)} \psi\left(\omega, t, X^{*}(\omega, t), \int_{\Omega} \phi_{\psi}\left(\omega^{\prime}, t, X^{*}\left(\omega^{\prime}, t\right), u^{*}\left(\omega^{\prime}, t\right)\right) \mathbb{P}\left(\mathrm{d} \omega^{\prime}\right), u^{*}(\omega, t),\right. \\
\zeta^{(r, \epsilon)}(\omega, t), \int_{\Omega} \partial \phi_{\psi}\left(\omega^{\prime}, t, X^{*}\left(\omega^{\prime}, t\right), u^{*}\left(\omega^{\prime}, t\right)\right) \zeta^{(r, \epsilon)}\left(\omega^{\prime}, t\right) \mathbb{P}\left(\mathrm{d} \omega^{\prime}\right), \\
\left.\int_{\Omega} \partial^{(2)} \phi_{\psi}\left(\omega^{\prime}, t, X^{*}\left(\omega^{\prime}, t\right), u^{*}\left(\omega^{\prime}, t\right)\right) \zeta^{(r, \epsilon)}\left(\omega^{\prime}, t\right)^{2} \mathbb{P}\left(\mathrm{d} \omega^{\prime}\right)\right)
\end{gathered}
$$

for all $\psi \in\left\{b, \sigma, f_{1}, \ldots, f_{N}\right\}$.

$$
\begin{aligned}
& \partial g_{i}(\omega):=\partial g_{i}\left(\omega, X^{*}(\omega, T), \int_{\Omega} \phi_{g_{i}}\left(\omega^{\prime}, X^{*}\left(\omega^{\prime}, T\right)\right) \mathbb{P}\left(\mathrm{d} \omega^{\prime}\right), \zeta^{(r, \epsilon)}(\omega, T),\right. \\
& \left.\int_{\Omega} \partial \phi_{g_{i}}\left(\omega^{\prime}, X^{*}\left(\omega^{\prime}, T\right)\right) \zeta^{(r, \epsilon)}\left(\omega^{\prime}, T\right) \mathbb{P}\left(\mathrm{d} \omega^{\prime}\right)\right) \\
& \partial^{(2)} g_{i}(\omega):=\partial^{(2)} g_{i}\left(\omega, X^{*}(\omega, T), \int_{\Omega} \phi_{g_{i}}\left(\omega^{\prime}, X^{*}\left(\omega^{\prime}, T\right)\right) \mathbb{P}\left(\mathrm{d} \omega^{\prime}\right), \zeta^{(r, \epsilon)}(\omega, T),\right. \\
& \left.\int_{\Omega} \partial \phi_{g_{i}}\left(\omega^{\prime}, X^{*}\left(\omega^{\prime}, T\right)\right) \zeta^{(r, \epsilon)}\left(\omega^{\prime}, T\right) \mathbb{P}\left(\mathrm{d} \omega^{\prime}\right), \int_{\Omega} \partial^{(2)} \phi_{g_{i}}\left(\omega^{\prime}, X^{*}\left(\omega^{\prime}, T\right)\right) \zeta^{(r, \epsilon)}\left(\omega^{\prime}, T\right)^{2} \mathbb{P}\left(\mathrm{d} \omega^{\prime}\right)\right)
\end{aligned}
$$


for all $i \in\{1, \ldots, N\}$.

$$
\begin{aligned}
& \Delta^{(r, \epsilon)} \psi(\omega, t):=\psi\left(\omega, t, X^{(r, \epsilon)}(\omega, t), \int_{\Omega} \phi_{\psi}\left(\omega^{\prime}, t, X^{(r, \epsilon)}\left(\omega^{\prime}, t\right), u_{i}^{*}\left(u_{i}\right)\left(\omega^{\prime}, t\right)\right) \mathbb{P}\left(\mathrm{d} \omega^{\prime}\right), u_{i}^{*}\left(u_{i}\right)(\omega, t)\right) \\
& -\psi\left(\omega, t, X^{*}(\omega, t), \int_{\Omega} \phi_{\psi}\left(\omega^{\prime}, t, X^{*}\left(\omega^{\prime}, t\right), u^{*}\left(\omega^{\prime}, t\right)\right) \mathbb{P}\left(\mathrm{d} \omega^{\prime}\right), u^{*}(\omega, t)\right) \\
& \delta^{(r, \epsilon)} \psi(\omega, t):=\psi\left(\omega, t, X^{(r, \epsilon)}(\omega, t), \int_{\Omega} \phi_{\psi}\left(\omega^{\prime}, t, X^{(r, \epsilon)}\left(\omega^{\prime}, t\right), u_{i}^{*}\left(u_{i}\right)\left(\omega^{\prime}, t\right)\right) \mathbb{P}\left(\mathrm{d} \omega^{\prime}\right), u_{i}^{*}\left(u_{i}\right)(\omega, t)\right) \\
& -\psi\left(\omega, t, X^{*}(\omega, t), \int_{\Omega} \phi_{\psi}\left(\omega^{\prime}, t, X^{*}\left(\omega^{\prime}, t\right), u_{i}^{*}\left(u_{i}\right)\left(\omega^{\prime}, t\right)\right) \mathbb{P}\left(\mathrm{d} \omega^{\prime}\right), u_{i}^{*}\left(u_{i}\right)(\omega, t)\right) \\
& \delta^{*} \psi(\omega, t):=\psi\left(\omega, t, X^{*}(\omega, t), \int_{\Omega} \phi_{\psi}\left(\omega^{\prime}, t, X^{*}\left(\omega^{\prime}, t\right), u_{i}^{*}\left(u_{i}\right)\left(\omega^{\prime}, t\right)\right) \mathbb{P}\left(\mathrm{d} \omega^{\prime}\right), u_{i}^{*}\left(u_{i}\right)(\omega, t)\right) \\
& -\psi\left(\omega, t, X^{*}(\omega, t), \int_{\Omega} \phi_{\psi}\left(\omega^{\prime}, t, X^{*}\left(\omega^{\prime}, t\right), u^{*}\left(\omega^{\prime}, t\right)\right) \mathbb{P}\left(\mathrm{d} \omega^{\prime}\right), u^{*}(\omega, t)\right)
\end{aligned}
$$

for all $\psi \in\left\{b, \sigma, f_{1}, \ldots, f_{N}\right\}$.

The families of all processes $\eta_{1}^{(r, \epsilon)}, \eta_{2}^{(r, \epsilon)}, \eta_{4, i}^{(r, \epsilon)}$, and random variables $\eta_{3, i}^{(r, \epsilon)}$ are defined as in Lemma A.1.

\section{Step 1. Decomposition.}

By the definition of the MFBSDE (4), the result of Lemma A.1, and the Itô product formula then

$$
\begin{aligned}
p_{i}(T) \zeta^{(r, \epsilon)}(T)=p_{i}(r & +\epsilon) \zeta^{(r, \epsilon)}(r+\epsilon)+\int_{r+\epsilon}^{T}\left(p_{i}(s)\left(\partial b(s)+\frac{1}{2} \partial^{(2)} b(s)+\eta_{1}^{(r, \epsilon)}(s)\right)\right. \\
& +q_{i}(s)\left(\partial \sigma(s)+\frac{1}{2} \partial^{(2)} \sigma(s)+\eta_{2}^{(r, \epsilon)}(s)\right) \\
& \left.-\zeta^{(r, \epsilon)}(s) \int_{\Omega} h_{i}\left(\omega^{\prime}, s, p_{i}(s), p_{i}\left(\omega^{\prime}, s\right), q_{i}(s), q_{i}\left(\omega^{\prime}, s\right)\right) \mathbb{P}\left(\mathrm{d} \omega^{\prime}\right)\right) \mathrm{d} s \\
& +\int_{r+\epsilon}^{T}\left\{p_{i}(s)\left(\partial \sigma(s)+\frac{1}{2} \partial^{(2)} \sigma(s)+\eta_{2}^{(r, \epsilon)}(s)\right)+q_{i}(s) \zeta^{(r, \epsilon)}(s)\right\} \mathrm{d} B(s)
\end{aligned}
$$

hence it follows, given the definition of the MFBSDE (4), that

$$
\begin{aligned}
\mathbb{E}_{\mathbb{P}}\left[\int_{r+\epsilon}^{T} \partial f_{i}(s) \mathrm{d} s+\partial g_{i}\right]=\mathbb{E}_{\mathbb{P}}\left[\int_{r+\epsilon}^{T} \partial f_{i}(s) \mathrm{d} s+p_{i}(T) \zeta^{(r, \epsilon)}(T)\right] \\
=\mathbb{E}_{\mathbb{P}}\left[p_{i}(r+\epsilon) \zeta^{(r, \epsilon)}(r+\epsilon)+\int_{r+\epsilon}^{T} \zeta^{(r, \epsilon)}(s)\left\{\partial_{x} f_{i}(s)+\partial_{x} b(s) p_{i}(s)+\partial_{x} \sigma(s) q_{i}(s)\right.\right. \\
\quad+\int_{\Omega}\left(\partial \phi_{f_{i}}(s) \partial_{y} f_{i}\left(\omega^{\prime}, s\right)+\partial \phi_{b}(s) \partial_{y} b\left(\omega^{\prime}, s\right) p_{i}\left(\omega^{\prime}, s\right)+\partial \phi_{\sigma}(s) \partial_{y} \sigma\left(\omega^{\prime}, s\right) q_{i}\left(\omega^{\prime}, s\right)\right. \\
\left.\left.\quad-h_{i}\left(\omega^{\prime}, s, p_{i}(s), p_{i}\left(\omega^{\prime}, s\right), q_{i}(s), q_{i}\left(\omega^{\prime}, s\right)\right)\right) \mathbb{P}\left(\mathrm{d} \omega^{\prime}\right)\right\} \mathrm{d} s \\
\left.\quad+\int_{r+\epsilon}^{T}\left(p_{i}(s)\left(\frac{1}{2} \partial^{(2)} b(s)+\eta_{1}^{(r, \epsilon)}(s)\right)+q_{i}(s)\left(\frac{1}{2} \partial^{(2)} \sigma(s)+\eta_{2}^{(r, \epsilon)}(s)\right)\right) \mathrm{d} s\right] \\
=\mathbb{E}_{\mathbb{P}}\left[p_{i}(r+\epsilon) \zeta^{(r, \epsilon)}(r+\epsilon)+\int_{r+\epsilon}^{T}\left(p_{i}(s)\left(\frac{1}{2} \partial^{(2)} b(s)+\eta_{1}^{(r, \epsilon)}(s)\right)+q_{i}(s)\left(\frac{1}{2} \partial^{(2)} \sigma(s)+\eta_{2}^{(r, \epsilon)}(s)\right)\right) \mathrm{d} s\right]
\end{aligned}
$$

for all $r \in[0, T)$ and $\epsilon \in(0, T-r]$.

Define, for each $r \in[0, T)$, the functions $I_{1}^{(r)}, I_{2}^{(r)}, I_{3}^{(r)}, I_{4}^{(r)}:(0, T-r] \rightarrow \mathbb{R}$ by

$$
\begin{aligned}
I_{1}^{(r)}(\epsilon) & :=\mathbb{E}_{\mathbb{P}}\left[\int_{r}^{r+\epsilon} \Delta^{(r, \epsilon)} f_{i}(s) \mathrm{d} s\right] \\
I_{2}^{(r)}(\epsilon) & :=\mathbb{E}_{\mathbb{P}}\left[p_{i}(r+\epsilon) \zeta^{(r, \epsilon)}(r+\epsilon)\right] \\
I_{3}^{(r)}(\epsilon) & :=\frac{1}{2} \mathbb{E}_{\mathbb{P}}\left[\int_{r+\epsilon}^{T}\left(p_{i}(s) \partial^{(2)} b(s)+q_{i}(s) \partial^{(2)} \sigma(s)+\partial^{(2)} f_{i}(s)\right) \mathrm{d} s+\partial^{(2)} g_{i}\right] \\
I_{4}^{(r)}(\epsilon) & :=\mathbb{E}_{\mathbb{P}}\left[\int_{r+\epsilon}^{T}\left(p_{i}(s) \eta_{1}^{(r, \epsilon)}(s)+q_{i}(s) \eta_{2}^{(r, \epsilon)}(s)+\eta_{4, i}^{(r, \epsilon)}(s)\right) \mathrm{d} s+\eta_{3, i}^{(r, \epsilon)}\right]
\end{aligned}
$$


for all $\epsilon \in(0, T-r]$, and so, from the above and Lemma A.1, then

$$
J_{i}\left(u_{i}^{*}\left(u_{i}^{(r, \epsilon)}\right)\right)-J_{i}\left(u^{*}\right)=I_{1}^{(r)}(\epsilon)+I_{2}^{(r)}(\epsilon)+I_{3}^{(r)}(\epsilon)+I_{4}^{(r)}(\epsilon)
$$

for all $r \in[0, T)$ and $\epsilon \in(0, T-r]$.

Step 2. Limit of $\epsilon^{-1} I_{1}^{(r)}(\epsilon)$.

By Assumption 2.1 and Lemma A.1, for any $r \in[0, T)$ then

$$
\lim _{\epsilon \downarrow 0} \epsilon^{-1} \int_{r}^{r+\epsilon} \mathbb{E}_{\mathbb{P}}\left[\delta^{(r, \epsilon)} f_{i}(s)\right] \mathrm{d} s=0 .
$$

Hence, by the fundamental theorem of calculus for the Lebesgue integral,

$$
\lim _{\epsilon \downarrow 0} \epsilon^{-1} I_{1}^{(r)}(\epsilon)=\mathbb{E}_{\mathbb{P}}\left[\delta^{*} f_{i}(r)\right]
$$

for Leb-almost all $r \in[0, T]$.

Step 3. Limit of $\epsilon^{-1} I_{2}^{(r)}(\epsilon)$.

Using the Itô product formula, we have that

$$
\begin{aligned}
I_{2}^{(r)}(r)=\int_{r}^{r+\epsilon} & \mathbb{E}_{\mathbb{P}}\left[p_{i}(s) \Delta^{(r, \epsilon)} b(s)\right. \\
& \left.-\left(\int_{\Omega} h_{i}\left(\omega^{\prime}, s, p_{i}(s), p_{i}\left(\omega^{\prime}, s\right), q_{i}(s), q_{i}\left(\omega^{\prime}, s\right)\right) \mathbb{P}\left(\mathrm{d} \omega^{\prime}\right)\right) \zeta^{(r, \epsilon)}(s)+q_{i}(s) \Delta^{(r, \epsilon)} \sigma(s)\right] \mathrm{d} s
\end{aligned}
$$

for all $r \in[0, T)$ and $\epsilon \in(0, T-r]$. By the Cauchy-Schwarz inequality, Assumption 2.1, Lemma A.1, the properties of each $p_{i}, q_{i}$, and $h_{i}$, and the dominated convergence theorem, then

$$
\begin{aligned}
\lim _{\epsilon \downarrow 0} \epsilon^{-1} \int_{r}^{r+\epsilon} & \mid \mathbb{E}_{\mathbb{P}}\left[p_{i}(s) \delta^{(r, \epsilon)} b(s)\right. \\
& \left.-\left(\int_{\Omega} h_{i}\left(\omega^{\prime}, s, p_{i}(s), p_{i}\left(\omega^{\prime}, s\right), q_{i}(s), q_{i}\left(\omega^{\prime}, s\right)\right) \mathbb{P}\left(\mathrm{d} \omega^{\prime}\right)\right) \zeta^{(r, \epsilon)}(s)+q_{i}(s) \delta^{(r, \epsilon)} \sigma(s)\right] \mid=0
\end{aligned}
$$

for any $r \in[0, T)$. Therefore, by the fundamental theorem of calculus for the Lebesgue integral,

$$
\lim _{\epsilon \downarrow 0} \epsilon^{-1} I_{2}^{(r)}(\epsilon)=\lim _{\epsilon \downarrow 0} \epsilon^{-1} \int_{r}^{r+\epsilon} \mathbb{E}_{\mathbb{P}}\left[p_{i}(s) \delta^{*} b(s)+q_{i}(s) \delta^{*} \sigma(s)\right] \mathrm{d} s=\mathbb{E}_{\mathbb{P}}\left[p_{i}(r) \delta^{*} b(r)+q_{i}(r) \delta^{*} \sigma(r)\right]
$$

for Leb-almost all $r \in[0, T]$.

\section{Step 4. Limit of $\epsilon^{-1} K_{1}^{(r)}(\epsilon)$ : Part 1 .}

Let

$$
I_{3}^{(r)}(\epsilon)=K_{1}^{(r)}(\epsilon)+K_{2}^{(r)}(\epsilon)
$$

for

$$
\begin{aligned}
K_{1}^{(r)}(\epsilon):=\frac{1}{2} \mathbb{E}_{\mathbb{P}} & {\left[\int _ { r + \epsilon } ^ { T } \left\{-Z^{(r, \epsilon)}(s)+\left(\partial_{x, x}^{(2)} f_{i}(s)+\mathbb{E}_{\mathbb{P}}\left[\partial_{y} f_{i}(s)\right] \partial^{(2)} \phi_{f_{i}}(s)\right.\right.\right.} \\
& +p_{i}(s) \partial_{x, x}^{(2)} b(s)+\mathbb{E}_{\mathbb{P}}\left[p_{i}(s) \partial_{y} b(s)\right] \partial^{(2)} \phi_{b}(s) \\
& \left.\left.+\partial_{x, x}^{(2)} \sigma(s) q_{i}(s)+\mathbb{E}_{\mathbb{P}}\left[\partial_{y} \sigma(s) q_{i}(s)\right] \partial^{(2)} \phi_{\sigma}(s)\right) \Xi^{(r, \epsilon)}(s)\right\} \mathrm{d} s \\
& \left.+\left(\partial_{x, x}^{(2)} g_{i}+\mathbb{E}_{\mathbb{P}}\left[\partial_{y} g_{i}\right] \partial^{(2)} \phi_{g_{i}}\right) \Xi^{(r, \epsilon)}(T)\right]
\end{aligned}
$$

and

$$
\begin{aligned}
K_{2}^{(r)}(\epsilon):=\frac{1}{2} & \int_{r+\epsilon}^{T}\left\{\mathbb{E}_{\mathbb{P}}\left[Z^{(r, \epsilon)}(s)\right]+\left(2 \mathbb{E}_{\mathbb{P}}\left[\zeta^{(r, \epsilon)}(s) p_{i}(s) \partial_{x, y}^{(2)} b(s)\right]\right.\right. \\
& \left.+\mathbb{E}_{\mathbb{P}}\left[\zeta^{(r, \epsilon)}(s) \partial \phi_{b}(s)\right] \mathbb{E}_{\mathbb{P}}\left[p_{i}(s) \partial_{y, y}^{(2)} b(s)\right]\right) \mathbb{E}_{\mathbb{P}}\left[\partial \phi_{b}(s) \zeta^{(r, \epsilon)}(s)\right] \\
& +\left(2 \mathbb{E}_{\mathbb{P}}\left[\zeta^{(r, \epsilon)}(s) \partial_{x, y}^{(2)} \sigma(s) q_{i}(s)\right]+\mathbb{E}_{\mathbb{P}}\left[\zeta^{(r, \epsilon)}(s) \partial \phi_{\sigma}(s)\right] \mathbb{E}_{\mathbb{P}}\left[\partial_{y, y}^{(2)} \sigma(s) q_{i}(s)\right]\right) \mathbb{E}_{\mathbb{P}}\left[\partial \phi_{\sigma}(s) \zeta^{(r, \epsilon)}(s)\right] \\
& \left.+\left(2 \mathbb{E}_{\mathbb{P}}\left[\zeta^{(r, \epsilon)}(s) \partial_{x, y}^{(2)} f_{i}(s)\right]+\mathbb{E}_{\mathbb{P}}\left[\zeta^{(r, \epsilon)}(s) \partial \phi_{f_{i}}(s)\right] \mathbb{E}_{\mathbb{P}}\left[\partial_{y, y}^{(2)} f_{i}(s)\right]\right) \mathbb{E}_{\mathbb{P}}\left[\partial \phi_{f_{i}}(s) \zeta^{(r, \epsilon)}(s)\right]\right\} \mathrm{d} s \\
& +\frac{1}{2}\left(2 \mathbb{E}_{\mathbb{P}}\left[\zeta^{(r, \epsilon)}(T) \partial_{x, y}^{(2)} g_{i}\right]+\mathbb{E}_{\mathbb{P}}\left[\zeta^{(r, \epsilon)}(T) \partial \phi_{g_{i}}\right] \mathbb{E}_{\mathbb{P}}\left[\partial_{y, y}^{(2)} g_{i}\right]\right) \mathbb{E}_{\mathbb{P}}\left[\partial \phi_{g_{i}} \zeta^{(r, \epsilon)}(T)\right]
\end{aligned}
$$


for all $r \in[0, T)$ and $\epsilon \in(0, T-r]$, where $\Xi^{(r, \epsilon)}:=\zeta^{(r, \epsilon)}(t)^{2}$ and $\left\{Z^{(r, \epsilon)}: \Omega \times[0, T] \rightarrow \mathbb{R} \mid r \in[0, T), \epsilon \in\right.$ $(0, T-r]\}$ is, for each $r \in[0, T)$ and $\epsilon \in(0, T-r]$, defined by

$$
\begin{aligned}
Z^{(r, \epsilon)}(t):= & P_{i}(t)\left(2 \zeta^{(r, \epsilon)}(t) \partial_{y} b(t) \mathbb{E}_{\mathbb{P}}\left[\partial \phi_{b}(t) \zeta^{(r, \epsilon)}(t)\right]+2 \partial_{x} \sigma(t) \zeta^{(r, \epsilon)}(t) \partial_{y} \sigma(t) \mathbb{E}_{\mathbb{P}}\left[\partial \phi_{\sigma}(t) \zeta^{(r, \epsilon)}(t)\right]\right. \\
& \left.+\left(\partial_{y} \sigma(t) \mathbb{E}_{\mathbb{P}}\left[\partial \phi_{\sigma}(t) \zeta^{(r, \epsilon)}(t)\right]\right)^{2}\right)+2 Q_{i}(t) \zeta^{(r, \epsilon)}(t) \partial_{y} \sigma(t) \mathbb{E}_{\mathbb{P}}\left[\partial \phi_{\sigma}(t) \zeta^{(r, \epsilon)}(t)\right]
\end{aligned}
$$

for $\mathbb{P}$-almost all $\omega \in \Omega$ uniformly for all $t \in[0, T]$.

Given the definition of the BSDE (5), one has, using the Itô product formula and Lemma A.1, that

$$
\begin{gathered}
\mathbb{E}_{\mathbb{P}}\left[\left(\partial_{x, x}^{(2)} g_{i}+\mathbb{E}_{\mathbb{P}}\left[\partial_{y} g_{i}\right] \partial^{(2)} \phi_{g_{i}}\right) \Xi^{(r, \epsilon)}(T)\right]=\mathbb{E}_{\mathbb{P}}\left[P_{i}(T) \Xi^{(r, \epsilon)}(T)\right] \\
=\mathbb{E}_{\mathbb{P}}\left[P_{i}(r+\epsilon) \Xi^{(r, \epsilon)}(r+\epsilon)\right]+\int_{r+\epsilon}^{T} \mathbb{E}_{\mathbb{P}}\left[R_{1}^{(r, \epsilon)}(s)-l_{i}\left(s, P_{i}(s), Q_{i}(s)\right) \Xi^{(r, \epsilon)}(s)\right. \\
\left.\quad+P_{i}(s)\left(2 \zeta^{(r, \epsilon)}(s) \partial b(s)+(\partial \sigma(s))^{2}\right)+2 Q_{i}(s) \zeta^{(r, \epsilon)}(s) \partial \sigma(s)\right] \mathrm{d} s
\end{gathered}
$$

where $\left\{R_{1}^{(r, \epsilon)}: \Omega \times[0, T] \rightarrow \mathbb{R} \mid r \in[0, T), \epsilon \in(0, T-r]\right\}$ is defined, for each $r \in[0, T)$ and $\epsilon \in(0, T-r]$, as

$$
\begin{aligned}
R_{1}^{(r, \epsilon)}(t):= & P_{i}(t)\left(2 \zeta^{(r, \epsilon)}(t)\left(\frac{1}{2} \partial^{(2)} b(t)+\eta_{1}^{(r, \epsilon)}(t)\right)+\left\{\left(\partial \sigma(t)+\frac{1}{2} \partial^{(2)} \sigma(t)+\eta_{2}^{(r, \epsilon)}(t)\right)^{2}-(\partial \sigma(t))^{2}\right\}\right) \\
& +2 Q_{i}(t) \zeta^{(r, \epsilon)}(t)\left(\frac{1}{2} \partial^{(2)} \sigma(t)+\eta_{2}^{(r, \epsilon)}(t)\right)
\end{aligned}
$$

for $\mathbb{P}$-almost all $\omega \in \Omega$ uniformly for all $t \in[0, T]$. Furthermore, it can be shown that

$$
\begin{array}{r}
\mathbb{E}_{\mathbb{P}}\left[P_{i}(T) \Xi^{(r, \epsilon)}(T)\right]=\mathbb{E}_{\mathbb{P}}\left[P_{i}(r+\epsilon) \Xi^{(r, \epsilon)}(r+\epsilon)+\int_{r+\epsilon}^{T}\left\{R_{1}^{(r, \epsilon)}(s)+Z^{(r, \epsilon)}(s)+\left(-l_{i}\left(s, P_{i}(s), Q_{i}(s)\right)\right.\right.\right. \\
\left.\left.\left.+2 P_{i}(s) \partial_{x} b(s)+P_{i}(s)\left(\partial_{x} \sigma(s)\right)^{2}+2 Q_{i}(s) \partial_{x} \sigma(s)\right) \Xi^{(r, \epsilon)}(s)\right\} \mathrm{d} s\right]
\end{array}
$$

and hence, given the definition of each $\ell_{i}$, then

$$
K_{1}^{(r)}(\epsilon)=\frac{1}{2} \mathbb{E}_{\mathbb{P}}\left[P_{i}(r+\epsilon) \Xi^{(r, \epsilon)}(r+\epsilon)+\int_{r+\epsilon}^{T} R_{1}^{(r, \epsilon)}(s) \mathrm{d} s\right]
$$

for all $r \in[0, T)$ and $\epsilon \in(0, T-r]$. Using Assumption 2.1, Lemma A.1 and the properties of each $P_{i}$ and $Q_{i}$, one may show that

$$
\lim _{\epsilon \downarrow 0} \epsilon^{-1} \mathbb{E}_{\mathbb{P}}\left[\int_{r+\epsilon}^{T} R_{1}^{(r, \epsilon)}(s) \mathrm{d} s\right]=0
$$

for all $r \in[0, T)$, and so

$$
\lim _{\epsilon \downarrow 0} \epsilon^{-1} K_{1}^{(r)}(\epsilon)=\lim _{\epsilon \downarrow 0} \frac{1}{2} \epsilon^{-1} \mathbb{E}_{\mathbb{P}}\left[P_{i}(r+\epsilon) \Xi^{(r, \epsilon)}(r+\epsilon)\right]
$$

for all $r \in[0, T)$.

Step 5. Limit of $\epsilon^{-1} K_{1}^{(r)}(\epsilon)$ : Part 2.

Using the Itô product formula, we have that

$$
\begin{aligned}
\mathbb{E}_{\mathbb{P}}\left[P_{i}(r+\epsilon) \Xi^{(r, \epsilon)}(r+\epsilon)\right]=\int_{r}^{r+\epsilon} \mathbb{E}_{\mathbb{P}}[ & P_{i}(s)\left(2 \zeta^{(r, \epsilon)}(s) \Delta^{(r, \epsilon)} b(s)+\left(\Delta^{(r, \epsilon)} \sigma(s)\right)^{2}\right) \\
& \left.-\ell_{i}\left(s, P_{i}(s), Q_{i}(s)\right) \Xi^{(r, \epsilon)}(s)+2 Q_{i}(s) \zeta^{(r, \epsilon)}(s) \Delta^{(r, \epsilon)} \sigma(s)\right] \mathrm{d} s
\end{aligned}
$$

for all $r \in[0, T)$ and $\epsilon \in(0, T-r]$. By the Cauchy-Schwarz inequality, Assumption 2.1, Lemma A.1, the properties of each $P_{i}, Q_{i}$, and $\ell_{i}$, Proposition 2.3, and the dominated convergence theorem, then

$$
\begin{aligned}
\lim _{\epsilon \downarrow 0} \epsilon^{-1} \int_{r}^{r+\epsilon} \mid \mathbb{E}_{\mathbb{P}}\left[P_{i}(s)\left(2 \zeta^{(r, \epsilon)}(s) \Delta^{(r, \epsilon)} b(s)+\left(\delta^{(r, \epsilon)} \sigma(s)\right)^{2}+2 \delta^{(r, \epsilon)} \sigma(s) \delta^{*} \sigma(s)\right)\right. \\
\left.-\ell_{i}\left(s, P_{i}(s), Q_{i}(s)\right) \Xi^{(r, \epsilon)}(s)+2 Q_{i}(s) \zeta^{(r, \epsilon)}(s) \Delta^{(r, \epsilon)} \sigma(s)\right] \mid \mathrm{d} s=0
\end{aligned}
$$


for all $r \in[0, T)$. Therefore, by the fundamental theorem of calculus for the Lebesgue integral,

$$
\lim _{\epsilon \downarrow 0} \epsilon^{-1} K_{1}^{(r)}(\epsilon)=\frac{1}{2} \lim _{\epsilon \downarrow 0} \epsilon^{-1} \int_{r}^{r+\epsilon} \mathbb{E}_{\mathbb{P}}\left[P_{i}(s)\left(\delta^{*} \sigma(s)\right)^{2}\right] \mathrm{d} s=\frac{1}{2} \mathbb{E}_{\mathbb{P}}\left[P_{i}(r)\left(\delta^{*} \sigma(r)\right)^{2}\right]
$$

for Leb-almost all $r \in[0, T]$.

Step 6. Limit of $\epsilon^{-1} K_{2}^{(r)}(\epsilon)$.

Let $(\bar{\Omega}, \overline{\mathcal{F}}, \overline{\mathbb{P}})$ be the completion of the product probability space $(\Omega \times \Omega, \mathcal{F} \times \mathcal{F}, \mathbb{P} \otimes \mathbb{P})$. Define the process $\bar{B}: \bar{\Omega} \times[0, T] \rightarrow \mathbb{R}^{2}$ by $\bar{B}(\bar{\omega}, t):=\left(B(\omega, t), B\left(\omega^{\prime}, t\right)\right)^{\top}$ for all $(\bar{\omega}, t)=\left(\left(\omega, \omega^{\prime}\right), t\right) \in \bar{\Omega} \times[0, T]$. Let $\overline{\mathbb{F}}:=\left\{\overline{\mathcal{F}}_{t} \mid t \in[0, T]\right\}$ be the $\overline{\mathbb{P}}$-augmented natural filtration generated by $\bar{B}$. Note that $\bar{B}$ is a $\mathbb{R}^{2}$-valued standard Brownian motion with respect to $\overline{\mathbb{F}}$ and which $\overline{\mathbb{P}}$-almost surely has continuous paths. Thus it is known that $\overline{\mathbb{F}}$ is right-continuous, and note that $\overline{\mathcal{F}}_{T}=\overline{\mathcal{F}}$.

Define the family of processes $\left\{\bar{\Xi}^{(r, \epsilon)}: \bar{\Omega} \times[0, T] \rightarrow \mathbb{R} \mid r \in[0, T), \epsilon \in(0, T-r]\right\}$ such that for each $r \in[0, T)$, and $\epsilon \in(0, T-r]$ then

$$
\bar{\Xi}^{(r, \epsilon)}(\bar{\omega}, t):=\zeta^{(r, \epsilon)}(\omega, t) \zeta^{(r, \epsilon)}\left(\omega^{\prime}, t\right)
$$

for $\overline{\mathbb{P}}$-almost all $\bar{\omega}=\left(\omega, \omega^{\prime}\right) \in \bar{\Omega}$ uniformly for all $t \in[0, T]$. Note that with respect to $(\bar{\Omega}, \overline{\mathcal{F}}, \overline{\mathbb{P}})$ and $\overline{\mathbb{F}}$, the quadratic-covariation of $(\bar{\omega}, t) \mapsto \zeta^{(r, \epsilon)}(\omega, t)$ and $(\bar{\omega}, t) \mapsto \zeta^{(r, \epsilon)}\left(\omega^{\prime}, t\right)$ is null. Hence, for each $r \in[0, T)$ and $\epsilon \in(0, T-r]$, by the Itô product formula then

$$
\bar{\Xi}^{(r, \epsilon)}(\bar{\omega}, t)=\int_{r}^{r \wedge t} \zeta^{(r, \epsilon)}(\omega, s) \mathrm{d} \zeta^{(r, \epsilon)}\left(\omega^{\prime}, s\right)+\int_{r}^{r \wedge t} \zeta^{(r, \epsilon)}\left(\omega^{\prime}, s\right) \mathrm{d} \zeta^{(r, \epsilon)}(\omega, s)
$$

for $\overline{\mathbb{P}}$-almost all $\bar{\omega} \in \bar{\Omega}$ uniformly for all $t \in[0, T]$.

Using the Tonelli-Fubini theorem (for completed product spaces), it can be shown that

$K_{2}^{(r)}(\epsilon)=\frac{1}{2} \int_{\bar{\Omega}} \int_{r+\epsilon}^{T} \Gamma(\bar{\omega}, s) \bar{\Xi}^{(r, \epsilon)}(\bar{\omega}, s) \mathrm{d} s+\left(2 \partial_{x, y}^{(2)} g_{i}(\omega)+\partial \phi_{g_{i}}(\omega) \mathbb{E}_{\mathbb{P}}\left[\partial_{y, y}^{(2)} g_{i}\right]\right) \partial \phi_{g_{i}}\left(\omega^{\prime}\right) \bar{\Xi}^{(r, \epsilon)}(\bar{\omega}, T) \overline{\mathbb{P}}(\mathrm{d} \bar{\omega})$

for all $r \in[0, T)$ and $\epsilon \in(0, T-r]$, where the process $\Gamma: \bar{\Omega} \times[0, T] \rightarrow \mathbb{R}$ is defined by

$$
\begin{aligned}
\Gamma(\bar{\omega}, t):= & 2 P_{i}(\omega, t) \partial_{y} b(\omega, t) \partial \phi_{b}\left(\omega^{\prime}, t\right)+2 P_{i}(\omega, t) \partial_{x} \sigma(\omega, t) \partial_{y} \sigma(\omega, t) \partial \phi_{\sigma}\left(\omega^{\prime}, t\right) \\
& +\mathbb{E}_{\mathbb{P}}\left[P_{i}(t)\left(\partial_{y} \sigma(t)\right)^{2}\right] \partial \phi_{\sigma}(\omega, t) \partial \phi_{\sigma}\left(\omega^{\prime}, t\right)+2 Q_{i}(\omega, t) \partial_{y} \sigma(\omega, t) \partial \phi_{\sigma}\left(\omega^{\prime}, t\right) \\
& +\left(2 p_{i}(\omega, t) \partial_{x, y}^{(2)} b(\omega, t)+\partial \phi_{b}(\omega, t) \mathbb{E}_{\mathbb{P}}\left[p_{i}(t) \partial_{y, y}^{(2)} b(t)\right]\right) \partial \phi_{b}\left(\omega^{\prime}, t\right) \\
& +\left(2 \partial_{x, y}^{(2)} \sigma(\omega, t) q_{i}(\omega, t)+\partial \phi_{\sigma}(\omega, t) \mathbb{E}_{\mathbb{P}}\left[\partial_{y, y}^{(2)} \sigma(t) q_{i}(t)\right]\right) \partial \phi_{\sigma}\left(\omega^{\prime}, t\right) \\
& +\left(2 \partial_{x, y}^{(2)} f_{i}(\omega, t)+\partial \phi_{f_{i}}(\omega, t) \mathbb{E}_{\mathbb{P}}\left[\partial_{y, y}^{(2)} f_{i}(t)\right]\right) \partial \phi_{f_{i}}\left(\omega^{\prime}, t\right)
\end{aligned}
$$

for $\overline{\mathbb{P}}$-almost all $\bar{\omega}=\left(\omega, \omega^{\prime}\right) \in \bar{\Omega}$ uniformly for all $t \in[0, T]$.

By Theorem 4.3 we may define the pair of processes $\left(\bar{P}_{i}, \bar{Q}_{i}\right) \in \mathcal{S}^{2}(\bar{\Omega} \times[0, T] ; \mathbb{R}) \times \mathcal{H}^{2}\left(\bar{\Omega} \times[0, T] ; \mathbb{R}^{2}\right)$ as the solution of the following CMFBSDE (see Section 4)

$$
\left\{\begin{aligned}
\bar{P}_{i}(\bar{\omega}, t)= & \bar{P}_{i}(\bar{\omega}, T)+\int_{t}^{T} \int_{\Omega} \bar{l}_{i}\left(\bar{\omega}, s, \omega^{\prime \prime}, \bar{P}_{i}(\bar{\omega}, s), \bar{P}_{i}\left(\left(\omega, \omega^{\prime \prime}\right), s\right), \bar{P}_{i}\left(\left(\omega^{\prime \prime}, \omega^{\prime}\right), s\right),\right. \\
& \left.\bar{Q}_{i}(\bar{\omega}, s), \bar{Q}_{i}\left(\left(\omega, \omega^{\prime \prime}\right), s\right), \bar{Q}_{i}\left(\left(\omega^{\prime \prime}, \omega^{\prime}\right), s\right)\right) \mathbb{P}\left(\mathrm{d} \omega^{\prime \prime}\right) \mathrm{d} s-\int_{t}^{T} \bar{Q}_{i}(\bar{\omega}, s)^{\top} \mathrm{d} \bar{B}(\bar{\omega}, s) \\
\bar{P}_{i}(\bar{\omega}, T)= & \left(2 \partial_{x, y}^{(2)} g_{i}(\omega)+\partial \phi_{g_{i}}(\omega) \mathbb{E}_{\mathbb{P}}\left[\partial_{y, y}^{(2)} g_{i}\right]\right) \partial \phi_{g_{i}}\left(\omega^{\prime}\right)
\end{aligned}\right.
$$

for $\overline{\mathbb{P}}$-almost all $\bar{\omega}=\left(\omega, \omega^{\prime}\right) \in \bar{\Omega}$ uniformly for all $t \in[0, T]$, where the generator $\bar{l}_{i}: \bar{\Omega} \times[0, T] \times \Omega \times \mathbb{R}^{9} \rightarrow$ $\mathbb{R}$ is defined as

$$
\begin{aligned}
\bar{l}_{i}\left(\bar{\omega}, t, \omega^{\prime \prime}, u, u^{\prime}, u^{\prime \prime}, v, v^{\prime}, v^{\prime \prime}\right):=\Gamma(\bar{\omega}, t) \\
+u\left(\partial_{x} b\left(\omega^{\prime}, t\right)+\partial_{x} b(\omega, t)\right)+u^{\prime} \partial_{y} b\left(\omega^{\prime \prime}, t\right) \partial \phi_{b}\left(\omega^{\prime}, t\right)+u^{\prime \prime} \partial_{y} b\left(\omega^{\prime \prime}, t\right) \partial \phi_{b}(\omega, t) \\
\quad+v_{1} \partial_{x} \sigma(\omega, t)+v_{2} \partial_{x} \sigma\left(\omega^{\prime}, t\right)+v_{2}^{\prime} \partial_{y} \sigma\left(\omega^{\prime \prime}, t\right) \partial \phi_{\sigma}\left(\omega^{\prime}, t\right)+v_{1}^{\prime \prime} \partial_{y} \sigma\left(\omega^{\prime \prime}, t\right) \partial \phi_{\sigma}(\omega, t)
\end{aligned}
$$

for all $\bar{\omega}=\left(\omega, \omega^{\prime}\right) \in \Omega_{0}, t \in[0, T], \omega^{\prime \prime} \in \Omega_{1}, u, u^{\prime}, u^{\prime \prime} \in \mathbb{R}$ and $v, v^{\prime}, v^{\prime \prime} \in \mathbb{R}^{2}$, for some $\Omega_{0} \subseteq \bar{\Omega}$ and $\Omega_{1} \subseteq \Omega$ with $\overline{\mathbb{P}}\left(\Omega_{0}\right)=\mathbb{P}\left(\Omega_{1}\right)=1$ such that the terms on the right-hand side of this definition are well-defined; and we set $\bar{l}_{i}\left(\bar{\omega}, t, \omega^{\prime \prime}, u, u^{\prime}, u^{\prime \prime}, v, v^{\prime}, v^{\prime \prime}\right)=0$ for all $\left(\bar{\omega}, t, \omega^{\prime \prime}, u, u^{\prime}, u^{\prime \prime}, v, v^{\prime}, v^{\prime \prime}\right)$ otherwise. 
Note that each $\bar{\ell}_{i}$ satisfies Condition 4.2. Given the definition of the CMFBSDE (9) then, by the Itô product formula, and Lemma A.1, it follows that

$$
\begin{aligned}
& \mathbb{E}_{\overline{\mathbb{P}}}\left[\bar{P}_{i}(T) \bar{\Xi}^{(r, \epsilon)}(T)\right]=\int_{\bar{\Omega}}\left\{\bar{P}_{i}(\bar{\omega}, r+\epsilon) \bar{\Xi}^{(r, \epsilon)}(\bar{\omega}, r+\epsilon)+\int_{r+\epsilon}^{T}\left\{R_{2}^{(r, \epsilon)}(\bar{\omega}, s)\right.\right. \\
&-\int_{\Omega} \bar{l}_{i}\left(\left(\omega, \omega^{\prime}, \omega^{\prime \prime}\right), s, \bar{P}_{i}(\bar{\omega}, s), \bar{P}_{i}\left(\left(\omega, \omega^{\prime \prime}\right), s\right), \bar{P}_{i}\left(\left(\omega^{\prime \prime}, \omega^{\prime}\right), s\right),\right. \\
&\left.\bar{Q}_{i}(\bar{\omega}, s), \bar{Q}_{i}\left(\left(\omega, \omega^{\prime \prime}\right), s\right), \bar{Q}_{i}\left(\left(\omega^{\prime \prime}, \omega^{\prime}\right), s\right)\right) \mathbb{P}\left(\mathrm{d} \omega^{\prime \prime}\right) \bar{\Xi}^{(r, \epsilon)}(\bar{\omega}, s) \\
&+\bar{P}_{i}(\bar{\omega}, s)\left(\zeta^{(r, \epsilon)}(\omega, s) \partial b\left(\omega^{\prime}, s\right)+\partial b(\omega, s) \zeta^{(r, \epsilon)}\left(\omega^{\prime}, s\right)\right) \\
&\left.\left.+\bar{Q}_{i}(\bar{\omega}, s)_{1} \partial \sigma(\omega, s) \zeta^{(r, \epsilon)}\left(\omega^{\prime}, s\right)+\bar{Q}_{i}(\bar{\omega}, s)_{2} \zeta^{(r, \epsilon)}(\omega, s) \partial \sigma\left(\omega^{\prime}, s\right)\right\} \mathrm{d} s\right\} \overline{\mathbb{P}}(\mathrm{d} \bar{\omega})
\end{aligned}
$$

where the family of processes $\left\{R_{2}^{(r, \epsilon)}: \bar{\Omega} \times[0, T] \rightarrow \mathbb{R} \mid r \in[0, T), \epsilon \in(0, T-r]\right\}$ is defined, for each $r \in[0, T)$ and $\epsilon \in(0, T-r]$, by

$$
\begin{aligned}
R_{2}^{(r, \epsilon)}(\bar{\omega}, t):= & \bar{P}_{i}(\bar{\omega}, t)\left(\zeta^{(r, \epsilon)}(\omega, t)\left(\frac{1}{2} \partial^{(2)} b\left(\omega^{\prime}, t\right)+\eta_{1}^{(r, \epsilon)}\left(\omega^{\prime}, t\right)\right)\right. \\
& \left.+\left(\frac{1}{2} \partial^{(2)} b(\omega, t)+\eta_{1}^{(r, \epsilon)}(\omega, t)\right) \zeta^{(r, \epsilon)}\left(\omega^{\prime}, t\right)\right) \\
& +\bar{Q}_{i}(\bar{\omega}, t)_{1}\left(\frac{1}{2} \partial^{(2)} \sigma(\omega, t)+\eta_{2}^{(r, \epsilon)}(\omega, t)\right) \zeta^{(r, \epsilon)}\left(\omega^{\prime}, t\right) \\
& +\bar{Q}_{i}(\bar{\omega}, t)_{2} \zeta^{(r, \epsilon)}(\omega, t)\left(\frac{1}{2} \partial^{(2)} \sigma\left(\omega^{\prime}, t\right)+\eta_{2}^{(r, \epsilon)}\left(\omega^{\prime}, t\right)\right)
\end{aligned}
$$

for $\overline{\mathbb{P}} \otimes$ Leb-almost all $(\bar{\omega}, t)=\left(\left(\omega, \omega^{\prime}\right), t\right) \in \bar{\Omega} \times[0, T]$. Furthermore, for each $r \in[0, T)$ and $\epsilon \in(0, T-r]$, it can be shown, using the Tonelli-Fubini theorem (for completed product spaces), that

$$
\begin{aligned}
\mathbb{E}_{\overline{\mathbb{P}}}\left[\bar{P}_{i}(T) \bar{\Xi}^{(r, \epsilon)}(T)\right]=\int_{\bar{\Omega}} & \left\{\bar{P}_{i}(\bar{\omega}, r+\epsilon) \bar{\Xi}^{(r, \epsilon)}(\bar{\omega}, r+\epsilon)+\int_{r+\epsilon}^{T}\left\{R_{2}^{(r, \epsilon)}(\bar{\omega}, s)+\int_{\Omega}(\right.\right. \\
& -\bar{l}_{i}\left(\left(\bar{\omega}, \omega^{\prime \prime}\right), s, \bar{P}_{i}(\bar{\omega}, s), \bar{P}_{i}\left(\left(\omega, \omega^{\prime \prime}\right), s\right), \bar{P}_{i}\left(\left(\omega^{\prime \prime}, \omega^{\prime}\right), s\right),\right. \\
& \left.\bar{Q}_{i}(\bar{\omega}, s), \bar{Q}_{i}\left(\left(\omega, \omega^{\prime \prime}\right), s\right), \bar{Q}_{i}\left(\left(\omega^{\prime \prime}, \omega^{\prime}\right), s\right)\right)+\bar{P}_{i}(\bar{\omega}, s)\left(\partial_{x} b\left(\omega^{\prime}, s\right)+\partial_{x} b(\omega, s)\right) \\
& +\partial_{y} b\left(\omega^{\prime \prime}, s\right)\left(\bar{P}_{i}\left(\left(\omega, \omega^{\prime \prime}\right), s\right) \partial \phi_{b}\left(\omega^{\prime}, s\right)+\bar{P}_{i}\left(\left(\omega^{\prime \prime}, \omega^{\prime}\right), s\right) \partial \phi_{b}(\omega, s)\right) \\
& +\bar{Q}_{i}(\bar{\omega}, s)_{1} \partial_{x} \sigma(\omega, s)+\bar{Q}_{i}(\bar{\omega}, s)_{2} \partial_{x} \sigma\left(\omega^{\prime}, s\right) \\
& \left.+\partial_{y} \sigma\left(\omega^{\prime \prime}, s\right)\left(\bar{Q}_{i}\left(\left(\omega^{\prime \prime}, \omega^{\prime}\right), s\right)_{1} \partial \phi_{\sigma}(\omega, s)+\bar{Q}_{i}\left(\left(\omega, \omega^{\prime \prime}\right), s\right)_{2} \partial \phi_{\sigma}\left(\omega^{\prime}, s\right)\right)\right) \mathbb{P}\left(\mathrm{d} \omega^{\prime \prime}\right) \\
& \} \bar{\Xi}^{(r, \epsilon)}(\bar{\omega}, s)\right\} \mathrm{d} s\right\} \overline{\mathbb{P}}(\mathrm{d} \bar{\omega}) .
\end{aligned}
$$

Given the definition of each $\bar{\ell}_{i}$, then

$$
K_{2}^{(r)}(\epsilon)=\frac{1}{2} \mathbb{E}_{\overline{\mathbb{P}}}\left[\bar{P}_{i}(r+\epsilon) \bar{\Xi}^{(r, \epsilon)}(r+\epsilon)+\int_{r+\epsilon}^{T} R_{2}^{(r, \epsilon)}(s) \mathrm{d} s\right]
$$

for all $r \in[0, T)$ and $\epsilon \in(0, T-r]$. Using Assumption 2.1, Lemma A.1, the Cauchy-Schwarz inequality, the Tonelli-Fubini theorem, and the properties of each $\bar{P}_{i}, \bar{Q}_{i}$, and $\bar{\ell}_{i}$, one may show that

$$
\lim _{\epsilon \downarrow 0} \epsilon^{-1} \mathbb{E}_{\overline{\mathbb{P}}}\left[\int_{r+\epsilon}^{T} R_{2}^{(r, \epsilon)}(s) \mathrm{d} s\right]=0
$$

for all $r \in[0, T)$. By the Itô product formula then

$$
\begin{aligned}
\mathbb{E}_{\overline{\mathbb{P}}}[ & \left.\bar{P}_{i}(r+\epsilon) \bar{\Xi}^{(r, \epsilon)}(r+\epsilon)\right] \\
= & \int_{\bar{\Omega}} \int_{r}^{r+\epsilon}\left\{-\int_{\Omega} \bar{l}_{i}\left(\left(\bar{\omega}, \omega^{\prime \prime}\right), s, \bar{P}_{i}(\bar{\omega}, s), \bar{P}_{i}\left(\left(\omega, \omega^{\prime \prime}\right), s\right), \bar{P}_{i}\left(\left(\omega^{\prime \prime}, \omega^{\prime}\right), s\right),\right.\right. \\
& \left.\bar{Q}_{i}(\bar{\omega}, s), \bar{Q}_{i}\left(\left(\omega, \omega^{\prime \prime}\right), s\right), \bar{Q}_{i}\left(\left(\omega^{\prime \prime}, \omega^{\prime}\right), s\right)\right) \mathbb{P}\left(\mathrm{d} \omega^{\prime \prime}\right) \bar{\Xi}^{(r, \epsilon)}(\bar{\omega}, s) \\
& +\bar{P}_{i}(\bar{\omega}, s)\left(\Delta^{(r, \epsilon)} b(\omega, s) \zeta^{(r, \epsilon)}\left(\omega^{\prime}, s\right)+\zeta^{(r, \epsilon)}(\omega, s) \Delta^{(r, \epsilon)} b\left(\omega^{\prime}, s\right)\right) \\
& \left.+\bar{Q}_{i}(\bar{\omega}, s)_{1} \Delta^{(r, \epsilon)} \sigma(\omega, s) \zeta^{(r, \epsilon)}\left(\omega^{\prime}, s\right)+\bar{Q}_{i}(\bar{\omega}, s)_{2} \zeta^{(r, \epsilon)}(\omega, s) \Delta^{(r, \epsilon)} \sigma\left(\omega^{\prime}, s\right)\right\} \mathrm{d} s \overline{\mathbb{P}}(\mathrm{d} \bar{\omega})
\end{aligned}
$$


for all $r \in[0, T)$ and $\epsilon \in(0, T-r]$. Therefore, using Assumption 2.1, Lemma A.1, Proposition 2.3, the Cauchy-Schwarz inequality, the Jensen inequality, the Tonelli-Fubini theorem, the dominated convergence theorem, and the fundamental theorem of calculus for the Lebesgue integral, then

$$
\lim _{\epsilon \downarrow 0} \epsilon^{-1} K_{2}^{(r)}(\epsilon)=0
$$

for Leb-almost all $r \in[0, T]$.

\section{Step 7.}

Recall that for each $r \in[0, T)$ then

$$
I_{4}^{(r)}(\epsilon):=\mathbb{E}_{\mathbb{P}}\left[\int_{r}^{T}\left(p_{i}(s) \eta_{1}^{(r, \epsilon)}(s)+q_{i}(s) \eta_{2}^{(r, \epsilon)}(s)+\eta_{4, i}^{(r, \epsilon)}(s)\right) \mathrm{d} s+\eta_{3, i}^{(r, \epsilon)}\right]
$$

for all $\epsilon \in(0, T-r]$. Using Lemma A.1, then

$$
\lim _{\epsilon \downarrow 0} \epsilon^{-1} I_{4}^{(r)}(\epsilon)=0
$$

for all $r \in[0, T)$.

Therefore, from this and all of the previous steps, we have proved equation (7), and so for any $u_{i} \in \mathcal{A}_{i}$ then equation (8) holds for Leb-almost all $r \in[0, T]$. In order to establish the SMP of equation (6) from the expectation-level SMP of equation (8) we apply the form of measurable-selection based argument which appears in the proof of Theorem 4.1 in [12] - that is based on our understanding that the definition of the control " $\tilde{u}^{\circ}$ " in [12] should be corrected to $\tilde{u}^{\circ}:=\tilde{u} I_{\Delta^{c}}+u^{\circ} I_{\Delta}$ (with respect to the notation in [12]). The rest of this proof below is thus an adaption of that basic argument to our setting, which we detail here for the sake of completeness and clarity.

Although the $N$-tuple of admissible controls $u^{*} \in \prod_{\ell=1}^{N} \mathcal{A}_{\ell}$ is assumed to be a Nash equilibrium, we now also suppose that the SMP of equation (6) does not hold, that is we now also suppose that the subset $R \in \mathcal{P}\left(\mathbb{G}_{i}\right)$ defined by

$$
R:=\left\{(\omega, t) \in \Omega \times[0, T] \mid \sup _{v \in U_{i}} H_{i}(\omega, t, v)>H_{i}\left(\omega, t, u_{i}^{*}(\omega, t)\right)\right\}
$$

is such that $\mathbb{P} \otimes \operatorname{Leb}(R)>0$.

Define a sequence of subsets $\left\{R_{m} \mid m \in \mathbb{N}\right\} \subseteq \mathcal{P}\left(\mathbb{G}_{i}\right)$ by

$$
R_{m}:=\left\{(\omega, t) \in R \mid \sup _{v \in U_{i}} H_{i}(\omega, t, v)>H_{i}\left(\omega, t, u_{i}^{*}(\omega, t)\right)+m^{-1}\right\}
$$

for all $m \in \mathbb{N}$. Since $R_{m} \uparrow R$, as $m \rightarrow \infty$, then by the continuity of the measure $\mathbb{P} \otimes$ Leb there exists a $m_{0} \in \mathbb{N}$ and $\epsilon>0$ such that $\mathbb{P} \otimes \operatorname{Leb}\left(R_{m}\right)>\epsilon$ for all $m \geq m_{0}$. The fact that $R,\left\{R_{m} \mid m \in \mathbb{N}\right\} \subseteq \mathcal{P}\left(\mathbb{G}_{i}\right)$ follows given Assumption 3.5.

Define the function $\bar{H}_{i}: \Omega \times[0, T] \times U_{i} \rightarrow \mathbb{R}$ by

$$
\bar{H}_{i}\left(\omega, t, u_{i}\right):=H_{i}\left(\omega, t, u_{i}\right)-\sup _{v \in U_{i}} H_{i}(\omega, t, v)
$$

for all $\left(\omega, t, u_{i}\right) \in \Omega \times[0, T] \times U_{i}$. Let $\Pi: \Omega \times[0, T] \rightsquigarrow U_{i}$ be the set-valued map defined by

$$
\Pi(\omega, t):=\left\{u_{i} \in U_{i} \mid \bar{H}_{i}\left(\omega, t, u_{i}\right) \in\left[-m_{0}^{-1}, 0\right]\right\}
$$

for all $(\omega, t) \in \Omega \times[0, T]$. Note that for all $(\omega, t) \in \Omega \times[0, T]$ the set $\Pi(\omega, t)$ is closed since the map $u_{i} \mapsto \bar{H}_{i}\left(\omega, t, u_{i}\right)$ is continuous. Also, note that for any $(\omega, t) \in \Omega \times[0, T]$ then the set $\Pi(\omega, t)$ is non-empty, indeed if it were not true then there would exist a pair $(\omega, t) \in \Omega \times[0, T]$ such that for each $u_{i} \in U_{i}$ then either:

or

$$
H_{i}\left(\omega, t, u_{i}\right)>\sup _{v \in U_{i}} H_{i}(\omega, t, v)
$$

$$
\sup _{v \in U_{i}} H_{i}(\omega, t, v)>H_{i}\left(\omega, t, u_{i}\right)+m_{0}^{-1}
$$

holds. Which is a contradiction of the definition of the supremum.

It can be shown (by using the monotone class theorem) that for each subset $V \in \mathcal{P}\left(\mathbb{G}_{i}\right) \times \mathcal{B}\left(U_{i}\right)$ then

$$
\left\{(\omega, t) \in \Omega \times[0, T] \mid V_{(\omega, t)} \cap O \neq \emptyset\right\} \in \mathcal{P}\left(\mathbb{G}_{i}\right)
$$


for all open sets $O \in \mathcal{B}\left(U_{i}\right)$, where $V_{(\omega, t)}:=\left\{u \in U_{i} \mid(\omega, t, u) \in V\right\}$. Since, from Assumption 3.5, the subset $Z \subseteq \Omega \times[0, T] \times U_{i}$ defined by

$$
Z:=\left\{\left(\omega, t, u_{i}\right) \in \Omega \times[0, T] \times U_{i} \mid \bar{H}_{i}\left(\omega, t, u_{i}\right) \in\left[-m_{0}^{-1}, 0\right]\right\}
$$

is in $\mathcal{P}\left(\mathbb{G}_{i}\right) \times \mathcal{B}\left(U_{i}\right)$, and as $\Pi(\omega, t)=Z_{(\omega, t)}$ for all $(\omega, t) \in \Omega \times[0, T]$, then

$$
\{(\omega, t) \in \Omega \times[0, T] \mid \Pi(\omega, t) \cap O \neq \emptyset\} \in \mathcal{P}\left(\mathbb{G}_{i}\right)
$$

for all open sets $O \in \mathcal{B}\left(U_{i}\right)$. That is, the set-valued map $\Pi$ is weakly measurable with respect to $\mathcal{P}\left(\mathbb{G}_{i}\right)$.

Let $\pi: \Omega \times[0, T] \rightarrow U_{i}$ be a $\mathcal{P}\left(\mathbb{G}_{i}\right)$-measurable selection for $\Pi$, in the sense that:

(i) $\pi$ is $\mathcal{P}\left(\mathbb{G}_{i}\right) \mid \mathcal{B}\left(U_{i}\right)$-measurable; and

(ii) $\pi(\omega, t) \in \Pi(\omega, t)$ for all $(\omega, t) \in \Omega \times[0, T]$.

Note that the existence of such a $\pi$ is given by the Kuratowski-Ryll-Nardzewski measurable section theorem (see [13]). Define the $\mathcal{A}_{i}$-admissible control $\bar{u}: \Omega \times[0, T] \rightarrow U_{i}$ by

$$
\bar{u}(\omega, t):=\left\{\begin{array}{cl}
\pi(\omega, t) & , \text { if }(\omega, t) \in R_{m_{0}} \\
u_{i}^{*}(\omega, t) & , \text { otherwise. }
\end{array}\right.
$$

First, note that

$$
\begin{aligned}
H_{i}(\omega, t, \bar{u}(\omega, t)) & =H_{i}(\omega, t, \pi(\omega, t)) \\
& \geq \sup _{v \in U_{i}} H_{i}(\omega, t, v)-m_{0}^{-1} \\
& >H_{i}\left(\omega, t, u_{i}^{*}(\omega, t)\right)
\end{aligned}
$$

for all $(\omega, t) \in R_{m_{0}}$. Secondly, it is clear that

$$
H_{i}(\omega, t, \bar{u}(\omega, t))=H_{i}\left(\omega, t, u_{i}^{*}(\omega, t)\right)
$$

for all $(\omega, t) \in(\Omega \times[0, T]) \backslash R_{m_{0}}$. Therefore

$$
\begin{aligned}
& \int_{0}^{T} \mathbb{E}_{\mathbb{P}}\left[H_{i}(t, \bar{u}(t))-H_{i}\left(t, u_{i}^{*}(t)\right)\right] \mathrm{d} t \\
& \quad=\int_{R_{m_{0}}}\left(H_{i}(\omega, t, \bar{u}(\omega, t))-H_{i}\left(\omega, t, u_{i}^{*}(\omega, t)\right)\right) \mathbb{P} \otimes \operatorname{Leb}(\mathrm{d} \omega, \mathrm{d} t)>0
\end{aligned}
$$

recalling that, from assumption, $\mathbb{P} \otimes \operatorname{Leb}\left(R_{m_{0}}\right)>0$. However, we know from before that if $u^{*}$ is a Nash equilibrium then one would have that

$$
\mathbb{E}_{\mathbb{P}}\left[H_{i}(t, \bar{u}(t))-H_{i}\left(t, u_{i}^{*}(t)\right)\right] \leq 0
$$

for Leb-almost all $t \in[0, T]$, which is a contradiction of equation (10). Thus-under the assumption that $u^{*}$ is a Nash equilibrium - the supposed property that $\mathbb{P} \otimes \operatorname{Leb}(R)>0$, has been shown to be false. That is, if $u^{*}$ is a Nash equilibrium then it must be that

$$
\max _{v \in U_{i}} H_{i}(\omega, t, v)=H_{i}\left(\omega, t, u_{i}^{*}(\omega, t)\right)
$$

for $\mathbb{P} \otimes$ Leb-almost all $(\omega, t) \in \Omega \times[0, T]$.

\section{Conditional mean-field BSDEs}

In this section we present an existence and uniqueness result for a class of backward stochastic equations that we call conditional mean-field backward stochastic differential equations (CMFBSDEs). This result ensures, in particular, that each of the adjoint processes $\left(\bar{P}_{i}, \bar{Q}_{i}\right)$ in Step 6 of the proof of Theorem 3.6 are well-defined as the solutions to certain such CMFBSDEs.

Let $(\bar{\Omega}, \overline{\mathcal{F}}, \overline{\mathbb{P}})$ be the completion of the product probability space $(\Omega \times \Omega, \mathcal{F} \times \mathcal{F}, \mathbb{P} \otimes \mathbb{P})$. Suppose that $B$ is now a standard $\mathbb{R}^{d}$-valued Brownian motion, with $\mathbb{P}$-almost sure continuous paths, and let $\mathbb{F}:=$ 
$\left\{\mathcal{F}_{t} \mid t \in[0, T]\right\}$ represent the corresponding $\mathbb{P}$-augmented natural filtration (which is known to be rightcontinuous). Assume that $\mathcal{F}_{T}=\mathcal{F}$. Define $\bar{B}: \bar{\Omega} \times[0, T] \rightarrow \mathbb{R}^{2 d}$ by $\bar{B}(\bar{\omega}, t):=\left(B(\omega, t)^{\top}, B\left(\omega^{\prime}, t\right)^{\top}\right)^{\top}$ for all $(\bar{\omega}, t)=\left(\left(\omega, \omega^{\prime}\right), t\right) \in \bar{\Omega} \times[0, T]$. Let $\overline{\mathbb{F}}:=\left\{\overline{\mathcal{F}}_{t} \mid t \in[0, T]\right\}$ be the $\overline{\mathbb{P}}$-augmented natural filtration generated by $\bar{B}$. Note that $\bar{B}$ is a $\mathbb{R}^{2 d}$-valued standard Brownian motion with respect to $\overline{\mathbb{F}}$, and which $\overline{\mathbb{P}}$-almost surely has continuous paths. Thus it is known that $\overline{\mathbb{F}}$ is right-continuous, and note that $\overline{\mathcal{F}}=\overline{\mathcal{F}}_{T}$.

Definition 4.1. Let $E$ be a finite dimensional Banach space with norm $\|\cdot\|_{E}$. For each $(\tilde{\Omega}, \tilde{\mathcal{F}}, \tilde{\mathbb{F}}, \tilde{\mathbb{P}})=$ $(\Omega, \mathcal{F}, \mathbb{F}, \mathbb{P}),(\bar{\Omega}, \overline{\mathcal{F}}, \overline{\mathbb{F}}, \overline{\mathbb{P}})$ then let:

- $\mathcal{H}^{2}(\tilde{\Omega} \times[0, T] ; E)$ denote the Hilbert space of $\tilde{\mathbb{P}} \otimes$ Leb-almost sure equivalence classes of $\mathcal{P}(\tilde{\mathbb{F}}) \mid \mathcal{B}(E)$ measurable processes $\phi: \tilde{\Omega} \times[0, T] \rightarrow E$ such that

$$
\|\phi\|_{\mathcal{H}^{2}(\tilde{\Omega} \times[0, T] ; E)}:=\left\|\left(\int_{0}^{T}\|\phi(t)\|_{E}^{2} \mathrm{~d} t\right)^{1 / 2}\right\|_{L^{2}(\tilde{\Omega}, \tilde{\mathcal{F}}, \tilde{\mathbb{P}} ; \mathbb{R})}<\infty
$$

- $\mathcal{S}^{2}(\tilde{\Omega} \times[0, T] ; E)$ denote the Banach space of $\tilde{\mathbb{P}}$-almost sure equivalence classes of random variables $\psi: \tilde{\Omega} \rightarrow \mathcal{C}([0, T] ; E)$ such that

$$
\|\psi\|_{\mathcal{S}^{2}(\tilde{\Omega} \times[0, T] ; E)}:=\left\|\sup _{t \in[0, T]}\right\| \psi(t)\left\|_{E}\right\|_{L^{2}(\tilde{\Omega}, \tilde{\mathcal{F}}, \tilde{\mathbb{P}} ; \mathbb{R})}<\infty
$$

and which, as processes, are $\tilde{\mathbb{F}}$-adapted (here $\mathcal{C}([0, T] ; E)$ denotes the space of all continuous functions with domain $[0, T]$ and range $E)$.

Condition 4.2. A function $l: \bar{\Omega} \times[0, T] \times \Omega \times \mathbb{R}^{3 n} \times\left(\mathbb{R}^{n} \otimes \mathbb{R}^{2 d}\right)^{3} \rightarrow \mathbb{R}^{n}$ is said to satisfy Condition 4.2 if the following properties hold:

- $\ell$ is $\mathcal{P}(\overline{\mathbb{F}}) \times \mathcal{F}_{T} \times \mathcal{B}\left(\mathbb{R}^{3 n} \times\left(\mathbb{R}^{n} \otimes \mathbb{R}^{2 d}\right)^{3}\right) \mid \mathcal{B}\left(\mathbb{R}^{n}\right)$-measurable;

- there exist subsets $\Omega_{0} \subseteq \bar{\Omega}$ and $\Omega_{1} \subseteq \Omega$, with $\overline{\mathbb{P}}\left(\Omega_{0}\right)=\mathbb{P}\left(\Omega_{1}\right)=1$, and a constant $c>0$ such that

$$
\begin{aligned}
\left\|l\left(\bar{\omega}, t, \omega^{\prime \prime}, x_{1}, x_{2}, x_{3}, y_{1}, y_{2}, y_{3}\right)-l\left(\bar{\omega}, t, \omega^{\prime \prime}, x_{1}^{\prime}, x_{2}^{\prime}, x_{3}^{\prime}, y_{1}^{\prime}, y_{2}^{\prime}, y_{3}^{\prime}\right)\right\|_{\mathbb{R}^{n}} & \\
& \leq c \sum_{i=1}^{3}\left(\left\|x_{i}-x_{i}^{\prime}\right\|_{\mathbb{R}^{n}}+\left\|y_{i}-y_{i}^{\prime}\right\|_{\mathbb{R}^{n} \otimes \mathbb{R}^{2 d}}\right)
\end{aligned}
$$

for all $\bar{\omega} \in \Omega_{0}, t \in[0, T], \omega^{\prime \prime} \in \Omega_{1}, x_{1}, x_{2}, x_{3} \in \mathbb{R}^{n}$ and $y_{1}, y_{2}, y_{3} \in \mathbb{R}^{n} \otimes \mathbb{R}^{2 d} ;$ and

- the process given by the mapping $(\bar{\omega}, t) \mapsto \int_{\Omega} l\left(\bar{\omega}, t, \omega^{\prime \prime}, 0,0,0,0,0,0\right) \mathbb{P}\left(\mathrm{d} \omega^{\prime \prime}\right)$ is in $\mathcal{H}^{2}(\bar{\Omega} \times$ $\left.[0, T] ; \mathbb{R}^{n}\right)$.

Theorem 4.3 (cf. Theorem 3.1 in [7]). Let $l: \bar{\Omega} \times[0, T] \times \Omega \times \mathbb{R}^{3 n} \times\left(\mathbb{R}^{n} \otimes \mathbb{R}^{2 d}\right)^{3} \rightarrow \mathbb{R}^{n}$ be a function satisfying Condition 4.2, and let $F \in L^{2}\left(\bar{\Omega}, \overline{\mathcal{F}}_{T}, \overline{\mathbb{P}} ; \mathbb{R}^{n}\right)$. There exists a unique $(Y, Z) \in$ $\mathcal{S}^{2}\left(\bar{\Omega} \times[0, T] ; \mathbb{R}^{n}\right) \times \mathcal{H}^{2}\left(\bar{\Omega} \times[0, T] ; \mathbb{R}^{n} \otimes \mathbb{R}^{2 d}\right)$ which solves the conditional mean-field backward stochastic differential equation

$$
\begin{aligned}
Y(\bar{\omega}, t)=F(\bar{\omega})+\int_{t}^{T} & \int_{\Omega} l\left(\bar{\omega}, s, \omega^{\prime \prime}, Y(\bar{\omega}, s), Y\left(\left(\omega, \omega^{\prime \prime}\right), s\right), Y\left(\left(\omega^{\prime \prime}, \omega^{\prime}\right), s\right),\right. \\
& \left.Z(\bar{\omega}, s), Z\left(\left(\omega, \omega^{\prime \prime}\right), s\right), Z\left(\left(\omega^{\prime \prime}, \omega^{\prime}\right), s\right)\right) \mathbb{P}\left(\mathrm{d} \omega^{\prime \prime}\right) \mathrm{d} s-\int_{t}^{T} Z(\bar{\omega}, s) \mathrm{d} \bar{B}(\bar{\omega}, s)
\end{aligned}
$$

for $\overline{\mathbb{P}}$-almost all $\bar{\omega}=\left(\omega, \omega^{\prime}\right) \in \bar{\Omega}$ uniformly for all $t \in[0, T]$, where given any $\left(Y^{\prime}, Z^{\prime}\right) \in \mathcal{H}^{2}(\bar{\Omega} \times$ $\left.[0, T] ; \mathbb{R}^{n}\right) \times \mathcal{H}^{2}\left(\bar{\Omega} \times[0, T] ; \mathbb{R}^{n} \otimes \mathbb{R}^{2 d}\right)$ then the function

$$
(\bar{\omega}, t, y, z) \mapsto \int_{\Omega} l\left(\bar{\omega}, t, \omega^{\prime \prime}, y, Y^{\prime}\left(\left(\omega, \omega^{\prime \prime}\right), t\right), Y^{\prime}\left(\left(\omega^{\prime \prime}, \omega^{\prime}\right), t\right), z, Z^{\prime}\left(\left(\omega, \omega^{\prime \prime}\right), t\right), Z^{\prime}\left(\left(\omega^{\prime \prime}, \omega^{\prime}\right), t\right)\right) \mathbb{P}\left(\mathrm{d} \omega^{\prime \prime}\right)
$$

defined for $\overline{\mathbb{P}}$-almost all $\bar{\omega} \in \bar{\Omega}$ uniformly for all $(t, x, y) \in[0, T] \times \mathbb{R}^{n} \times \mathbb{R}^{n} \otimes \mathbb{R}^{2 d}$, is taken as denoting the corresponding $\mathcal{P}(\overline{\mathbb{F}}) \times \mathcal{B}\left(\mathbb{R}^{n} \times \mathbb{R}^{n} \otimes \mathbb{R}^{2 d}\right) \mid \mathcal{B}\left(\mathbb{R}^{n}\right)$-measurable function that is given by Lemma 4.4 .

Proof. We prove the theorem by slightly modifying the two step procedure used to prove Theorem 3.1 in [7] — which is an existence and uniqueness result for the solution of certain mean-field BSDEs.

Step 1. Fix any $\alpha \in \mathcal{H}^{2}\left(\bar{\Omega} \times[0, T] ; \mathbb{R}^{n}\right)$, and $\beta \in \mathcal{H}^{2}\left(\bar{\Omega} \times[0, T] ; \mathbb{R}^{n} \otimes \mathbb{R}^{2 d}\right)$. Define $l^{\alpha, \beta}$ : $\bar{\Omega} \times[0, T] \times \mathbb{R}^{n} \times \mathbb{R}^{n} \otimes \mathbb{R}^{2 d} \rightarrow \mathbb{R}^{n}$ by

$$
l^{\alpha, \beta}(\bar{\omega}, t, x, y):=\int_{\Omega} l\left(\bar{\omega}, t, \omega^{\prime \prime}, x, \alpha\left(\left(\omega, \omega^{\prime \prime}\right), t\right), \alpha\left(\left(\omega^{\prime \prime}, \omega^{\prime}\right), t\right), y, \beta\left(\left(\omega, \omega^{\prime \prime}\right), t\right), \beta\left(\left(\omega^{\prime \prime}, \omega^{\prime}\right), t\right)\right) \mathbb{P}\left(\mathrm{d} \omega^{\prime \prime}\right)
$$


for $\overline{\mathbb{P}}$-almost all $\bar{\omega}=\left(\omega, \omega^{\prime}\right) \in \bar{\Omega}$ uniformly for all $(t, x, y) \in[0, T] \times \mathbb{R}^{n} \times \mathbb{R}^{n} \otimes \mathbb{R}^{2 d}$, where this integral denotes the corresponding $\mathcal{P}(\overline{\mathbb{F}}) \times \mathcal{B}\left(\mathbb{R}^{n} \times \mathbb{R}^{n} \otimes \mathbb{R}^{2 d}\right) \mid \mathcal{B}\left(\mathbb{R}^{n}\right)$-measurable function given by Lemma 4.4. Given Condition 4.2 and Lemma 4.4 then note that:

- $l^{\alpha, \beta}$ is $\mathcal{P}(\overline{\mathbb{F}}) \times \mathcal{B}\left(\mathbb{R}^{n} \times \mathbb{R}^{n} \otimes \mathbb{R}^{2 d}\right) \mid \mathcal{B}\left(\mathbb{R}^{n}\right)$-measurable;

- there exists a constant $c>0$ such that

$$
\left\|l^{\alpha, \beta}(\bar{\omega}, t, x, y)-l^{\alpha, \beta}\left(\bar{\omega}, t, x^{\prime}, y^{\prime}\right)\right\|_{\mathbb{R}^{n}} \leq c\left(\left\|x-x^{\prime}\right\|_{\mathbb{R}^{n}}+\left\|y-y^{\prime}\right\|_{\mathbb{R}^{n} \otimes \mathbb{R}^{2 d}}\right)
$$

for $\overline{\mathbb{P}}$-almost all $\bar{\omega} \in \bar{\Omega}$ uniformly for all $t \in[0, T], x, x^{\prime} \in \mathbb{R}^{n}$ and $y, y^{\prime} \in \mathbb{R}^{n} \otimes \mathbb{R}^{2 d}$; and

- the process defined by $(\bar{\omega}, t) \mapsto l^{\alpha, \beta}(\bar{\omega}, t, 0,0)$ is in $\mathcal{H}^{2}\left(\bar{\Omega} \times[0, T] ; \mathbb{R}^{n}\right)$.

Therefore, for each $\alpha \in \mathcal{H}^{2}\left(\bar{\Omega} \times[0, T] ; \mathbb{R}^{n}\right)$, and $\beta \in \mathcal{H}^{2}\left(\bar{\Omega} \times[0, T] ; \mathbb{R}^{n} \otimes \mathbb{R}^{2 d}\right)$, given these above properties of $l^{\alpha, \beta}$, known results (see, for example, subsection 2.1 of [8]) imply that there exists a unique solution $\left(Y^{\alpha, \beta}, Z^{\alpha, \beta}\right) \in \mathcal{S}^{2}\left(\bar{\Omega} \times[0, T] ; \mathbb{R}^{n}\right) \times \mathcal{H}^{2}\left(\bar{\Omega} \times[0, T] ; \mathbb{R}^{n} \otimes \mathbb{R}^{2 d}\right)$ to the BSDE

$$
Y^{\alpha, \beta}(\bar{\omega}, t)=F(\bar{\omega})+\int_{t}^{T} l^{\alpha, \beta}\left(\bar{\omega}, s, Y^{\alpha, \beta}(\bar{\omega}, s), Z^{\alpha, \beta}(\bar{\omega}, s)\right) \mathrm{d} s-\int_{t}^{T} Z^{\alpha, \beta}(\bar{\omega}, s) \mathrm{d} \bar{B}(\bar{\omega}, s)
$$

for $\overline{\mathbb{P}}$-almost all $\bar{\omega} \in \bar{\Omega}$ uniformly for all $t \in[0, T]$. In particular it can be shown that this uniqueness of the solution is such that if $(\alpha, \beta)=\left(\alpha^{\prime}, \beta^{\prime}\right)$ in $\mathcal{H}^{2}\left(\bar{\Omega} \times[0, T] ; \mathbb{R}^{n}\right) \times \mathcal{H}^{2}\left(\bar{\Omega} \times[0, T] ; \mathbb{R}^{n} \otimes \mathbb{R}^{2 d}\right)$ then the respective solutions must be equal $\left(Y^{\alpha, \beta}, Z^{\alpha, \beta}\right)=\left(Y^{\alpha^{\prime}, \beta^{\prime}}, Z^{\alpha^{\prime}, \beta^{\prime}}\right)$ in $\mathcal{S}^{2}\left(\bar{\Omega} \times[0, T] ; \mathbb{R}^{n}\right) \times \mathcal{H}^{2}(\bar{\Omega} \times$ $\left.[0, T] ; \mathbb{R}^{n} \otimes \mathbb{R}^{2 d}\right)$.

Step 2. Let $\gamma \in(0, \infty)$ be some constant that will be specified later. By the Itô formula and the definition of the $\operatorname{BSDE}(12)$, for any $\alpha, \alpha^{\prime} \in \mathcal{H}^{2}\left(\bar{\Omega} \times[0, T] ; \mathbb{R}^{n}\right)$, and $\beta, \beta^{\prime} \in \mathcal{H}^{2}\left(\bar{\Omega} \times[0, T] ; \mathbb{R}^{n} \otimes \mathbb{R}^{2 d}\right)$ one has that

$$
\begin{aligned}
0= & e^{\gamma T}\left\|Y^{\alpha, \beta}(T)-Y^{\alpha^{\prime}, \beta^{\prime}}(T)\right\|_{\mathbb{R}^{n}}^{2} \\
= & \left\|Y^{\alpha, \beta}(0)-Y^{\alpha^{\prime}, \beta^{\prime}}(0)\right\|_{\mathbb{R}^{n}}^{2}+\mathbb{E}_{\overline{\mathbb{P}}}\left[\int_{0}^{T} \gamma e^{\gamma s}\left\|Y^{\alpha, \beta}(s)-Y^{\alpha^{\prime}, \beta^{\prime}}(s)\right\|_{\mathbb{R}^{n}}^{2} \mathrm{~d} s\right. \\
& \quad+\int_{0}^{T} e^{\gamma s}\left\|Z^{\alpha, \beta}(s)-Z^{\alpha^{\prime}, \beta^{\prime}}(s)\right\|_{\mathbb{R}^{n} \otimes \mathbb{R}^{2 d}}^{2} \mathrm{~d} s+2 \int_{0}^{T} e^{\gamma s}\left\langle Y^{\alpha, \beta}(s)-Y^{\alpha^{\prime}, \beta^{\prime}}(s),\right. \\
& \left.\left.l^{\alpha^{\prime}, \beta^{\prime}}\left(s, Y^{\alpha^{\prime}, \beta^{\prime}}(s), Z^{\alpha^{\prime}, \beta^{\prime}}(s)\right)-l^{\alpha, \beta}\left(s, Y^{\alpha, \beta}(s), Z^{\alpha, \beta}(s)\right)\right\rangle_{\mathbb{R}^{n}} \mathrm{~d} s\right]
\end{aligned}
$$

and so

$$
\begin{aligned}
& \mathbb{E}_{\overline{\mathbb{P}}} {\left[\int_{0}^{T} \gamma e^{\gamma s}\left\|Y^{\alpha, \beta}(s)-Y^{\alpha^{\prime}, \beta^{\prime}}(s)\right\|_{\mathbb{R}^{n}}^{2} \mathrm{~d} s\right]+\mathbb{E}_{\overline{\mathbb{P}}}\left[\int_{0}^{T} e^{\gamma s}\left\|Z^{\alpha, \beta}(s)-Z^{\alpha^{\prime}, \beta^{\prime}}(s)\right\|_{\mathbb{R}^{n} \otimes \mathbb{R}^{2 d}}^{2} \mathrm{~d} s\right] } \\
& \leq 2 \mathbb{E}_{\overline{\mathbb{P}}}\left[\int _ { 0 } ^ { T } e ^ { \gamma s } \left\langleY^{\alpha, \beta}(s)-Y^{\alpha^{\prime}, \beta^{\prime}}(s),\right.\right. \\
&\left.\left.\quad l^{\alpha, \beta}\left(s, Y^{\alpha, \beta}(s), Z^{\alpha, \beta}(s)\right)-l^{\alpha^{\prime}, \beta^{\prime}}\left(s, Y^{\alpha^{\prime}, \beta^{\prime}}(s), Z^{\alpha^{\prime}, \beta^{\prime}}(s)\right)\right\rangle_{\mathbb{R}^{n}} \mathrm{~d} s\right] .
\end{aligned}
$$

By the Cauchy-Schwarz inequality, the assumed Lipschitz property of $l$, and Lemma 4.4 , there exists a constant $c>0$ (independent of $\gamma$ ) such that

$$
\begin{aligned}
& \mathbb{E}_{\overline{\mathbb{P}}}\left[\int_{0}^{T} \gamma e^{\gamma s}\left\|Y^{\alpha, \beta}(s)-Y^{\alpha^{\prime}, \beta^{\prime}}(s)\right\|_{\mathbb{R}^{n}}^{2} \mathrm{~d} s\right]+\mathbb{E}_{\overline{\mathbb{P}}}\left[\int_{0}^{T} e^{\gamma s}\left\|Z^{\alpha, \beta}(s)-Z^{\alpha^{\prime}, \beta^{\prime}}(s)\right\|_{\mathbb{R}^{n} \otimes \mathbb{R}^{2 d}}^{2} \mathrm{~d} s\right] \\
& \leq 2 c \int_{\bar{\Omega}}\left[\int _ { 0 } ^ { T } e ^ { \gamma s } \| Y ^ { \alpha , \beta } ( \overline { \omega } , s ) - Y ^ { \alpha ^ { \prime } , \beta ^ { \prime } } ( \overline { \omega } , s ) \| _ { \mathbb { R } ^ { n } } \left(\left\|Y^{\alpha, \beta}(\bar{\omega}, s)-Y^{\alpha^{\prime}, \beta^{\prime}}(\bar{\omega}, s)\right\|_{\mathbb{R}^{n}}\right.\right. \\
& +\left\|Z^{\alpha, \beta}(\bar{\omega}, s)-Z^{\alpha^{\prime}, \beta^{\prime}}(\bar{\omega}, s)\right\|_{\mathbb{R}^{n} \otimes \mathbb{R}^{2 d}}+\int_{\Omega}\left\{\left\|\alpha\left(\left(\omega, \omega^{\prime \prime}\right), s\right)-\alpha^{\prime}\left(\left(\omega, \omega^{\prime \prime}\right), s\right)\right\|_{\mathbb{R}^{n}}\right. \\
& +\left\|\alpha\left(\left(\omega^{\prime \prime}, \omega^{\prime}\right), s\right)-\alpha^{\prime}\left(\left(\omega^{\prime \prime}, \omega^{\prime}\right), s\right)\right\|_{\mathbb{R}^{n}}+\left\|\beta\left(\left(\omega, \omega^{\prime \prime}\right), s\right)-\beta^{\prime}\left(\left(\omega, \omega^{\prime \prime}\right), s\right)\right\|_{\mathbb{R}^{n} \otimes \mathbb{R}^{2 d}} \\
& \left.\left.\left.+\left\|\beta\left(\left(\omega^{\prime \prime}, \omega^{\prime}\right), s\right)-\beta^{\prime}\left(\left(\omega^{\prime \prime}, \omega^{\prime}\right), s\right)\right\|_{\mathbb{R}^{n} \otimes \mathbb{R}^{2 d}}\right\} \mathbb{P}\left(\mathrm{d} \omega^{\prime \prime}\right)\right) \mathrm{d} s\right] \overline{\mathbb{P}}(\mathrm{d} \bar{\omega}) .
\end{aligned}
$$

Moreover, using the Tonelli-Fubini theorem, the arithmetic-geometric mean inequality, and Jensen's 
inequality, it follows that there exists a constant $c>0$ (independent of $\gamma$ ) such that

$$
\begin{aligned}
& \left(\gamma-c\left(2+\lambda^{2}\right)\right) \mathbb{E}_{\overline{\mathbb{P}}}\left[\int_{0}^{T} e^{\gamma s}\left\|Y^{\alpha, \beta}(s)-Y^{\alpha^{\prime}, \beta^{\prime}}(s)\right\|_{\mathbb{R}^{n}}^{2} \mathrm{~d} s\right] \\
& +\left(1-\frac{c}{\lambda^{2}}\right) \mathbb{E}_{\overline{\mathbb{P}}}\left[\int_{0}^{T} e^{\gamma s}\left\|Z^{\alpha, \beta}(s)-Z^{\alpha^{\prime}, \beta^{\prime}}(s)\right\|_{\mathbb{R}^{n} \otimes \mathbb{R}^{2 d}}^{2} \mathrm{~d} s\right] \\
& \quad \leq \frac{2 c}{\lambda^{2}} \mathbb{E}_{\overline{\mathbb{P}}}\left[\int_{0}^{T} e^{\gamma s}\left(\left\|\alpha(s)-\alpha^{\prime}(s)\right\|_{\mathbb{R}^{n}}^{2}+\left\|\beta(s)-\beta^{\prime}(s)\right\|_{\mathbb{R}^{n} \otimes \mathbb{R}^{2 d}}^{2}\right) \mathrm{d} s\right]
\end{aligned}
$$

for all $\lambda \in \mathbb{R}$.

Define a norm $\|\cdot\|_{\gamma}$ on $H^{2}:=\mathcal{H}^{2}\left(\bar{\Omega} \times[0, T] ; \mathbb{R}^{n}\right) \oplus \mathcal{H}^{2}\left(\bar{\Omega} \times[0, T] ; \mathbb{R}^{n} \otimes \mathbb{R}^{2 d}\right)$ by

$$
\|(Y, Z)\|_{\gamma}:=\mathbb{E}_{\overline{\mathbb{P}}}\left[\int_{0}^{T} e^{\gamma s}\left(\|Y(s)\|_{\mathbb{R}^{n}}^{2}+\|Z(s)\|_{\mathbb{R}^{n} \otimes \mathbb{R}^{2 d}}^{2}\right) \mathrm{d} s\right]^{1 / 2}
$$

for all $(Y, Z) \in H^{2}$. Let $S^{2}:=\mathcal{S}^{2}\left(\bar{\Omega} \times[0, T] ; \mathbb{R}^{n}\right) \oplus \mathcal{H}^{2}\left(\bar{\Omega} \times[0, T] ; \mathbb{R}^{n} \otimes \mathbb{R}^{2 d}\right)$ be the direct sum Banach space with norm

$$
\|(Y, Z)\|_{S^{2}}:=\left(\|Y\|_{\mathcal{S}^{2}\left(\bar{\Omega} \times[0, T] ; \mathbb{R}^{n}\right)}^{2}+\|Z\|_{\mathcal{H}^{2}\left(\bar{\Omega} \times[0, T] ; \mathbb{R}^{n} \otimes \mathbb{R}^{2 d}\right)}^{2}\right)^{1 / 2}
$$

for all $(Y, Z) \in S^{2}$. By Step 1, one may define a mapping $I: H^{2} \rightarrow S^{2}$ as that which takes each $(\alpha, \beta) \in H^{2}$ to the solution $\left(Y^{\alpha, \beta}, Z^{\alpha, \beta}\right)$ of equation (12). Recall that by the uniqueness property noted in Step 1, if $(\alpha, \beta)=\left(\alpha^{\prime}, \beta^{\prime}\right)$ in $H^{2}$ then $I(\alpha, \beta)=I\left(\alpha^{\prime}, \beta^{\prime}\right)$ in $S^{2}$.

Given the constant $c>0$ in equation (13), by setting $\lambda=\sqrt{8 c}$ and $\gamma=2 c(1+4 c)+0.75$ then equation (13) implies that

$$
\left\|I(\alpha, \beta)-I\left(\alpha^{\prime}, \beta^{\prime}\right)\right\|_{\gamma} \leq \frac{1}{3}\left\|(\alpha, \beta)-\left(\alpha^{\prime}, \beta^{\prime}\right)\right\|_{\gamma}
$$

for all $(\alpha, \beta),\left(\alpha^{\prime}, \beta^{\prime}\right) \in H^{2}$. By the Banach contraction mapping theorem there exists a unique $\left(\alpha^{*}, \beta^{*}\right) \in H^{2}$ such that $I\left(\alpha^{*}, \beta^{*}\right)=\left(\alpha^{*}, \beta^{*}\right)$ in $H^{2}$.

Let $\alpha^{* *} \in \mathcal{S}^{2}\left(\bar{\Omega} \times[0, T] ; \mathbb{R}^{n}\right)$ be defined by $\alpha^{* *}:=Y^{\alpha^{*}, \beta^{*}}$. Note that $\left(\alpha^{* *}, \beta^{*}\right)=\left(\alpha^{*}, \beta^{*}\right)$ in $H^{2}$, and so $I\left(\alpha^{* *}, \beta^{*}\right)=I\left(\alpha^{*}, \beta^{*}\right)$ in $S^{2}$. Therefore

$$
I\left(\alpha^{* *}, \beta^{*}\right)=\left(\alpha^{* *}, \beta^{*}\right)
$$

in $S^{2}$, that is $\left(\alpha^{* *}, \beta^{*}\right)$ is a $\mathcal{S}^{2}\left(\bar{\Omega} \times[0, T] ; \mathbb{R}^{n}\right) \times \mathcal{H}^{2}\left(\bar{\Omega} \times[0, T] ; \mathbb{R}^{n} \otimes \mathbb{R}^{2 d}\right)$-solution of the CMFBSDE (11).

To establish the property of uniqueness, suppose that both pairs $(Y, Z)$ and $\left(Y^{\prime}, Z^{\prime}\right)$ are $\mathcal{S}^{2}(\bar{\Omega} \times$ $\left.[0, T] ; \mathbb{R}^{n}\right) \times \mathcal{H}^{2}\left(\bar{\Omega} \times[0, T] ; \mathbb{R}^{n} \otimes \mathbb{R}^{2 d}\right)$-solutions of the CMFBSDE (11). By the Minkowski, Jensen, and Cauchy-Schwarz inequalities, the Doob martingale inequality, the Itô-isometry, and Lemma 4.4, then there exists a constant $c>0$ such that

$$
\begin{gathered}
\left.\mathbb{E}_{\overline{\mathbb{P}}} \sup _{t \in[0, T]}\left\|Y(t)-Y^{\prime}(t)\right\|_{\mathbb{R}^{n}}^{2}\right] \leq c \int_{\bar{\Omega}} \int_{0}^{T}\left[T \int_{\Omega} \| l\left(\bar{\omega}, s, \omega^{\prime \prime}, Y(\bar{\omega}, s), Y\left(\left(\omega, \omega^{\prime \prime}\right), s\right),\right.\right. \\
\left.Y\left(\left(\omega^{\prime \prime}, \omega^{\prime}\right), s\right), Z(\bar{\omega}, s), Z\left(\left(\omega, \omega^{\prime \prime}\right), s\right), Z\left(\left(\omega^{\prime \prime}, \omega^{\prime}\right), s\right)\right) \\
-l\left(\bar{\omega}, s, \omega^{\prime \prime}, Y^{\prime}(\bar{\omega}, s), Y^{\prime}\left(\left(\omega, \omega^{\prime \prime}\right), s\right), Y^{\prime}\left(\left(\omega^{\prime \prime}, \omega^{\prime}\right), s\right),\right. \\
\left.Z^{\prime}(\bar{\omega}, s), Z^{\prime}\left(\left(\omega, \omega^{\prime \prime}\right), s\right), Z^{\prime}\left(\left(\omega^{\prime \prime}, \omega^{\prime}\right), s\right)\right) \|_{\mathbb{R}^{n}}^{2} \mathbb{P}\left(\mathrm{d} \omega^{\prime \prime}\right) \\
+\left\|Z(\bar{\omega}, s)-Z^{\prime}(\bar{\omega}, s)\right\|_{\left.\mathbb{R}^{n} \otimes \mathbb{R}^{2 d}\right]}^{2} \mathrm{~d} s \overline{\mathbb{P}}(\mathrm{d} \bar{\omega}) .
\end{gathered}
$$

Therefore, by the Lipschitz property of $l$ from Condition 4.2, and the Tonelli-Fubini theorem, there exists a constant $c>0$ such that

$$
\begin{aligned}
& \mathbb{E}_{\mathbb{P}}\left[\sup _{t \in[0, T]}\left\|Y(t)-Y^{\prime}(t)\right\|_{\mathbb{R}^{n}}^{2}\right] \\
& \leq c \int_{0}^{T}\left(\mathbb{E}_{\mathbb{P}}\left[\sup _{s \in[0, t]}\left\|Y(s)-Y^{\prime}(s)\right\|_{\mathbb{R}^{n}}^{2}\right]+\mathbb{E}_{\mathbb{P}}\left[\left\|Z(t)-Z^{\prime}(t)\right\|_{\left.\left.\mathbb{R}^{n} \otimes \mathbb{R}^{2 d}\right]\right) \mathrm{d} t}^{2}\right.\right.
\end{aligned}
$$


Any possible solution $\left(Y^{\prime \prime}, Z^{\prime \prime}\right) \in \mathcal{S}^{2}\left(\bar{\Omega} \times[0, T] ; \mathbb{R}^{n}\right) \times \mathcal{H}^{2}\left(\bar{\Omega} \times[0, T] ; \mathbb{R}^{n} \otimes \mathbb{R}^{2 d}\right)$ of the CMFBSDE (11) gives a unique fixed point of the map $I$ on $H^{2}$. Thus $(Y, Z)=\left(Y^{\prime}, Z^{\prime}\right)$ in $H^{2}$, and so equation (14) reduces to the fact that there exists a constant $c>0$ such that

$$
\mathbb{E}_{\mathbb{P}}\left[\sup _{t \in[0, T]}\left\|Y(t)-Y^{\prime}(t)\right\|_{\mathbb{R}^{n}}^{2}\right] \leq c \int_{0}^{T} \mathbb{E}_{\mathbb{P}}\left[\sup _{s \in[0, t]}\left\|Y(s)-Y^{\prime}(s)\right\|_{\mathbb{R}^{n}}^{2}\right] \mathrm{d} t
$$

then moreover by the Gronwall inequality it follows that $Y=Y^{\prime}$ in $\mathcal{S}^{2}\left(\bar{\Omega} \times[0, T] ; \mathbb{R}^{n}\right)$. Therefore the solution $(Y, Z) \in \mathcal{S}^{2}\left(\bar{\Omega} \times[0, T] ; \mathbb{R}^{n}\right) \times \mathcal{H}^{2}\left(\bar{\Omega} \times[0, T] ; \mathbb{R}^{n} \otimes \mathbb{R}^{2 d}\right)$ to the CMFBSDE (11) is unique.

Lemma 4.4. For any function $\ell: \bar{\Omega} \times[0, T] \times \Omega \times \mathbb{R}^{3 n} \times\left(\mathbb{R}^{n} \otimes \mathbb{R}^{2 d}\right)^{3} \rightarrow \mathbb{R}^{n}$ satisfying Condition 4.2, and any $(\alpha, \beta) \in \mathcal{H}^{2}\left(\bar{\Omega} \times[0, T] ; \mathbb{R}^{n}\right) \times \mathcal{H}^{2}\left(\bar{\Omega} \times[0, T] ; \mathbb{R}^{n} \otimes \mathbb{R}^{2 d}\right)$ then let the process $\ell^{\alpha, \beta}: \bar{\Omega} \times[0, T] \times$ $\mathbb{R}^{n} \times \mathbb{R}^{n} \otimes \mathbb{R}^{2 d} \rightarrow \mathbb{R}^{n}$ be defined by

$$
\ell^{\alpha, \beta}(\bar{\omega}, t, x, y):=\int_{\Omega} \ell\left(\bar{\omega}, t, \omega^{\prime \prime}, x, \alpha\left(\left(\omega, \omega^{\prime \prime}\right), t\right), \alpha\left(\left(\omega^{\prime \prime}, \omega^{\prime}\right), t\right), y, \beta\left(\left(\omega, \omega^{\prime \prime}\right), t\right), \beta\left(\left(\omega^{\prime \prime}, \omega^{\prime}\right), t\right)\right) \mathbb{P}\left(\mathrm{d} \omega^{\prime \prime}\right)
$$

for $\overline{\mathbb{P}} \otimes$ Leb-almost all $(\bar{\omega}, t) \in \bar{\Omega} \times[0, T]$ uniformly for all $(x, y) \in \mathbb{R}^{n} \times \mathbb{R}^{n} \otimes \mathbb{R}^{2 d}$. That is, we first define the integral for each fixed $(x, y) \in \mathbb{R}^{n} \times \mathbb{R}^{n} \otimes \mathbb{R}^{2 d}$ using the Tonelli-Fubini theorem for completed product spaces, so that the integral is well-defined for $\overline{\mathbb{P}} \otimes$ Leb-almost all $(\bar{\omega}, t) \in \bar{\Omega} \times[0, T]$ uniformly for all $(x, y) \in \mathbb{Q}^{n} \times \mathbb{Q}^{n} \otimes \mathbb{Q}^{2 d}$, then we may extend the definition to $\overline{\mathbb{P}} \otimes$ Leb-almost all $(\bar{\omega}, t) \in \bar{\Omega} \times[0, T]$ uniformly for all $(x, y) \in \mathbb{R}^{n} \times \mathbb{R}^{n} \otimes \mathbb{R}^{2 d}$ by using the dominated convergence theorem and Condition 4.2.

There exists a function $\bar{\ell}^{\alpha, \beta}: \bar{\Omega} \times[0, T] \times \mathbb{R}^{n} \times \mathbb{R}^{n} \otimes \mathbb{R}^{2 d} \rightarrow \mathbb{R}^{n}$ such that:

- $\bar{\ell}^{\alpha, \beta}(\bar{\omega}, t, x, y)=\ell^{\alpha, \beta}(\bar{\omega}, t, x, y)$ for $\overline{\mathbb{P}} \otimes$ Leb-almost all $(\bar{\omega}, t) \in \bar{\Omega} \times[0, T]$ uniformly for all $(x, y) \in$ $\mathbb{R}^{n} \times \mathbb{R}^{n} \otimes \mathbb{R}^{2 d} ;$ and

- $\bar{\ell}^{\alpha, \beta}$ is $\mathcal{P}(\overline{\mathbb{F}}) \times \mathcal{B}\left(\mathbb{R}^{n} \times \mathbb{R}^{n} \otimes \mathbb{R}^{2 d}\right) \mid \mathcal{B}\left(\mathbb{R}^{n}\right)$-measurable.

Proof. Step 1. For this first step of the proof we suppose that $\alpha$ and $\beta$ are elementary processes of the form

$$
\alpha(\bar{\omega}, t)=a_{0}(\bar{\omega})+\sum_{i=1}^{m} a_{i}(\bar{\omega}) \mathbf{1}_{\left(t_{i-1}, t_{i}\right]}(t), \quad \beta(\bar{\omega}, t)=b_{0}(\bar{\omega})+\sum_{i=1}^{m} b_{i}(\bar{\omega}) \mathbf{1}_{\left(t_{i-1}, t_{i}\right]}(t)
$$

for some $0=t_{0}<t_{1}<\ldots<t_{m}=T$, where for each $i \in\{0,1, \ldots, m\}$ then $a_{i}$ and $b_{i}$ are bounded and, respectively, $\overline{\mathcal{F}}_{t_{i-1}} \mid \mathcal{B}\left(\mathbb{R}^{n}\right)$-measurable and $\overline{\mathcal{F}}_{t_{i-1}} \mid \mathcal{B}\left(\mathbb{R}^{n} \otimes \mathbb{R}^{2 d}\right)$-measurable random variables, denoting $t_{-1}:=0$. Note that for each $t \in[0, T]$ then $\overline{\mathcal{F}}_{t}=\overline{\mathcal{F}}_{t} \times\left.\mathcal{F}_{t}\right|_{\overline{\mathbb{P}}}$, and as such it follows that for any $C \in \mathcal{B}\left(\mathbb{R}^{n}\right)$ then

$$
\begin{aligned}
\{(\bar{\omega}, t) \in \bar{\Omega} \times[0, T] \mid \alpha(\bar{\omega}, t) \in C\} & =\left(\left\{\bar{\omega} \mid a_{0}(\bar{\omega}) \in C\right\} \times\left\{t_{0}\right\}\right) \cup\left(\cup_{i=1}^{m}\left\{\bar{\omega} \mid a_{i}(\bar{\omega}) \in C\right\} \times\left(t_{i-1}, t_{i}\right]\right) \\
& =\left(A_{0} \cup B_{0} \times\left\{t_{0}\right\}\right) \cup\left(\cup_{i=1}^{m} A_{i} \cup B_{i} \times\left(t_{i-1}, t_{i}\right]\right)
\end{aligned}
$$

for some subsets $\left\{A_{i} \mid i \in\{0,1, \ldots, m\}\right\} \subseteq \bar{\Omega}$, where $A_{i} \in \mathcal{F}_{t_{i-1}} \times \mathcal{F}_{t_{i-1}}$ for each $i \in\{0,1, \ldots, m\}$, and $\left\{B_{i} \mid i \in\{0,1, \ldots, m\}\right\} \subseteq \mathcal{N}_{\overline{\mathbb{P}}}$, where $\mathcal{N}_{\overline{\mathbb{P}}}$ denotes the collection of all $\overline{\mathbb{P}}$-negligible subsets of $\bar{\Omega}$. For such subsets $\left\{A_{i} \mid i \in\{0,1, \ldots, m\}\right\}$ then it may be seen that

$$
\left\{\left(\omega, \omega^{\prime}, t, \omega^{\prime \prime}\right) \in \bar{\Omega} \times[0, T] \times \Omega \mid\left(\omega, \omega^{\prime \prime}, t\right) \in\left(A_{0} \times\left\{t_{0}\right\}\right) \cup\left(\cup_{i=1}^{m} A_{i} \times\left(t_{i-1}, t_{i}\right]\right)\right\} \in \mathcal{P}(\overline{\mathbb{F}}) \times \mathcal{F}_{T}
$$

and for such subsets $\left\{B_{i} \mid i \in\{0,1, \ldots, m\}\right\}$ then, by the Tonelli-Fubini theorem

$$
\left\{\left(\omega, \omega^{\prime}, t, \omega^{\prime \prime}\right) \in \bar{\Omega} \times[0, T] \times \Omega \mid\left(\omega, \omega^{\prime \prime}, t\right) \in\left(B_{0} \times\left\{t_{0}\right\}\right) \cup\left(\cup_{i=1}^{m} B_{i} \times\left(t_{i-1}, t_{i}\right]\right)\right\} \in \mathcal{T}
$$

where $\mathcal{T}$ denotes the collection of subsets defined by

$$
\begin{aligned}
& \mathcal{T}:=\left\{\left\{\left(\omega, \omega^{\prime}, t, \omega^{\prime \prime}\right) \mid\left(\omega, \omega^{\prime}, \omega^{\prime \prime}, t\right) \in \cup_{i=1}^{\ell} N_{i} \times M_{i}\right\} \mid\right. \\
& \left.\ell \in \mathbb{N},\left\{N_{i}\right\}_{i \in\{1, \ldots, \ell\}} \subseteq \mathcal{N}_{\mathbb{P} \otimes \mathbb{P} \otimes \mathbb{P}},\left\{M_{i}\right\}_{i \in\{1, \ldots, \ell\}} \subseteq \mathcal{B}([0, T])\right\}
\end{aligned}
$$


and where $\mathcal{N}_{\mathbb{P} \otimes \mathbb{P} \otimes \mathbb{P}}$ denotes the collection of all $\mathbb{P} \otimes \mathbb{P} \otimes \mathbb{P}$-negligible subsets of $\Omega \times \Omega \times \Omega$. Hence, using a similar argument for the process $\beta$, it follows that for any $C \in \mathcal{P}(\overline{\mathbb{F}}) \times \mathcal{F}_{T} \times \mathcal{B}\left(\mathbb{R}^{n} \times \mathbb{R}^{n} \otimes \mathbb{R}^{2 d}\right)$ then

$$
\begin{aligned}
\left\{\left(\bar{\omega}, t, \omega^{\prime \prime}, x, y\right)\right. & \in \bar{\Omega} \times[0, T] \times \Omega \times \mathbb{R}^{n} \times \mathbb{R}^{n} \otimes \mathbb{R}^{2 d} \mid \\
\left.\left(\bar{\omega}, t, \omega^{\prime \prime}, x, \alpha\left(\left(\omega, \omega^{\prime \prime}\right), t\right), \alpha\left(\left(\omega^{\prime \prime}, \omega^{\prime}\right), t\right), y, \beta\left(\left(\omega, \omega^{\prime \prime}\right), t\right), \beta\left(\left(\omega^{\prime \prime}, \omega^{\prime}\right), t\right)\right) \in C\right\} & \in \sigma\left(\mathcal{P}(\overline{\mathbb{F}}) \times \mathcal{F}_{T}, \mathcal{T}\right) \times \mathcal{B}\left(\mathbb{R}^{n} \times \mathbb{R}^{n} \otimes \mathbb{R}^{2 d}\right) .
\end{aligned}
$$

Let $\mathcal{M}$ be the family of subsets defined by

$$
\begin{aligned}
\mathcal{M} & :=\left\{M \in \sigma\left(\mathcal{P}(\overline{\mathbb{F}}) \times \mathcal{F}_{T}, \mathcal{T}\right) \times \mathcal{B}\left(\mathbb{R}^{n} \times \mathbb{R}^{n} \otimes \mathbb{R}^{2 d}\right) \mid\right. \text { the map } \\
(\bar{\omega}, t, x, y) & \left.\mapsto \int_{\Omega} \mathbf{1}_{\left\{\omega_{0} \in \Omega \mid\left(\bar{\omega}, t, \omega_{0}, x, y\right) \in M\right\}}\left(\omega^{\prime \prime}\right) \mathbb{P}\left(\mathrm{d} \omega^{\prime \prime}\right) \text { is } \mathcal{P}(\overline{\mathbb{F}}) \times \mathcal{B}\left(\mathbb{R}^{n} \times \mathbb{R}^{n} \otimes \mathbb{R}^{2 d}\right) \text {-measurable }\right\} .
\end{aligned}
$$

By the Tonelli-Fubini theorem then $\mathcal{P}(\overline{\mathbb{F}}) \times \mathcal{F}_{T} \times \mathcal{B}\left(\mathbb{R}^{n} \times \mathbb{R}^{n} \otimes \mathbb{R}^{2 d}\right) \subseteq \mathcal{M}$ and, also, for any set $M:=M_{1} \times M_{2}$, for some $M_{1} \in \mathcal{T}$ and $M_{2} \in \mathcal{B}\left(\mathbb{R}^{n} \times \mathbb{R}^{n} \otimes \mathbb{R}^{2 d}\right)$, then

$$
\int_{\Omega} \mathbf{1}_{\left\{\omega_{0} \in \Omega \mid\left(\bar{\omega}, t, \omega_{0}, x, y\right) \in M\right\}}\left(\omega^{\prime \prime}\right) \mathbb{P}\left(\mathrm{d} \omega^{\prime \prime}\right)=0
$$

for $\overline{\mathbb{P}}$-almost all $\bar{\omega} \in \bar{\Omega}$ uniformly for all $(t, x, y) \in[0, T] \times \mathbb{R}^{n} \times \mathbb{R}^{n} \otimes \mathbb{R}^{2 d}$, thus $M \in \mathcal{M}$. Moreover, it may be shown that $\mathcal{M}$ contains the algebra of subsets formed from the collection of all finite length compositions of the set operations union, intersection and complement and sets from the families $\mathcal{P}(\overline{\mathbb{F}}) \times \mathcal{F}_{T} \times \mathcal{B}\left(\mathbb{R}^{n} \times \mathbb{R}^{n} \otimes \mathbb{R}^{2 d}\right)$ and $\left\{M_{1} \times M_{2} \mid M_{1} \in \mathcal{T}, M_{2} \in \mathcal{B}\left(\mathbb{R}^{n} \times \mathbb{R}^{n} \otimes \mathbb{R}^{2 d}\right)\right\}$. Thus $\mathcal{M}$ contains the algebra of subsets generated by $\left\{\mathcal{P}(\overline{\mathbb{F}}) \times \mathcal{F}_{T} \times \mathcal{B}\left(\mathbb{R}^{n} \times \mathbb{R}^{n} \otimes \mathbb{R}^{2 d}\right),\left\{M_{1} \times M_{2} \mid M_{1} \in \mathcal{T}, M_{2} \in\right.\right.$ $\left.\left.\mathcal{B}\left(\mathbb{R}^{n} \times \mathbb{R}^{n} \otimes \mathbb{R}^{2 d}\right)\right\}\right\}$.

Let $\left\{S_{m}\right\}_{m \in \mathbb{N}} \subseteq \mathcal{M}$ be such that $S_{m} \subseteq S_{m+1}$ for each $m \in \mathbb{N}$. For each $m \in \mathbb{N} \cup\{\infty\}$ define the function $f_{m}: \bar{\Omega} \times[0, T] \times \mathbb{R}^{n} \times \mathbb{R}^{n} \otimes \mathbb{R}^{2 d} \rightarrow \mathbb{R}$ by

$$
f_{m}(\bar{\omega}, t, x, y):=\int_{\Omega} \mathbf{1}_{\left\{\omega_{0} \in \Omega \mid\left(\bar{\omega}, t, \omega_{0}, x, y\right) \in \cup_{i=1}^{m} S_{i}\right\}}\left(\omega^{\prime \prime}\right) \mathbb{P}\left(\mathrm{d} \omega^{\prime \prime}\right)
$$

for all $(\bar{\omega}, t, x, y) \in \bar{\Omega} \times[0, T] \times \mathbb{R}^{n} \times \mathbb{R}^{n} \otimes \mathbb{R}^{2 d}$. By the monotone convergence theorem then, for each $(\bar{\omega}, t, x, y) \in \bar{\Omega} \times[0, T] \times \mathbb{R}^{n} \times \mathbb{R}^{n} \otimes \mathbb{R}^{2 d}, f_{\infty}(\bar{\omega}, t, x, y)=\lim _{m \rightarrow \infty} f_{m}(\bar{\omega}, t, x, y)$ and so, given that for each $m \in \mathbb{N}$ then $f_{m}$ is $\mathcal{P}(\overline{\mathbb{F}}) \times \mathcal{B}\left(\mathbb{R}^{n} \times \mathbb{R}^{n} \otimes \mathbb{R}^{2 d}\right)$-measurable, it follows that $f_{\infty}$ is $\mathcal{P}(\overline{\mathbb{F}}) \times \mathcal{B}\left(\mathbb{R}^{n} \times \mathbb{R}^{n} \otimes \mathbb{R}^{2 d}\right)$ measurable and hence $\cup_{i=1}^{\infty} S_{i} \in \mathcal{M}$. Similarly, one may show that for any $\left\{S_{m}\right\}_{m \in \mathbb{N}} \subseteq \mathcal{M}$ such that $S_{m} \supseteq S_{m+1}$ for each $m \in \mathbb{N}$, then $\cap_{m=1}^{\infty} S_{m} \in \mathcal{M}$. Thus $\mathcal{M}$ is a monotone class, and so by the monotone class theorem $\mathcal{M}=\sigma\left(\mathcal{P}(\overline{\mathbb{F}}) \times \mathcal{F}_{T}, \mathcal{T}\right) \times \mathcal{B}\left(\mathbb{R}^{n} \times \mathbb{R}^{n} \otimes \mathbb{R}^{2 d}\right)$. Given equation (15), and the fact that $\ell$ is $\mathcal{P}(\overline{\mathbb{F}}) \times \mathcal{F}_{T} \times \mathcal{B}\left(\mathbb{R}^{3 n} \times\left(\mathbb{R}^{n} \otimes \mathbb{R}^{2 d}\right)^{3}\right) \mid \mathcal{B}\left(\mathbb{R}^{n}\right)$-measurable, then it follows that $\ell^{\alpha, \beta}$ is $\mathcal{P}(\overline{\mathbb{F}}) \times \mathcal{B}\left(\mathbb{R}^{n} \times \mathbb{R}^{n} \otimes \mathbb{R}^{2 d}\right) \mid \mathcal{B}\left(\mathbb{R}^{n}\right)$-measurable.

Step 2. For this second step of the proof we suppose that $\alpha \in \mathcal{H}^{2}\left(\bar{\Omega} \times[0, T] ; \mathbb{R}^{n}\right)$ and $\beta \in$ $\mathcal{H}^{2}\left(\bar{\Omega} \times[0, T] ; \mathbb{R}^{n} \otimes \mathbb{R}^{2 d}\right)$ are general such processes. It is known that there exist sequences of such elementary processes $\left\{\alpha_{m}\right\}_{m \in \mathbb{N}} \subseteq \mathcal{H}^{2}\left(\bar{\Omega} \times[0, T] ; \mathbb{R}^{n}\right)$ and $\left\{\beta_{m}\right\}_{m \in \mathbb{N}} \subseteq \mathcal{H}^{2}\left(\bar{\Omega} \times[0, T] ; \mathbb{R}^{n} \otimes \mathbb{R}^{2 d}\right)$ such that $\alpha_{m} \rightarrow_{m \rightarrow \infty} \alpha$ in $\mathcal{H}^{2}\left(\bar{\Omega} \times[0, T] ; \mathbb{R}^{n}\right)$, and $\beta_{m} \rightarrow_{m \rightarrow \infty} \beta$ in $\mathcal{H}^{2}\left(\bar{\Omega} \times[0, T] ; \mathbb{R}^{n} \otimes \mathbb{R}^{2 d}\right)$. Under Condition 4.2 , then there exists a constant $c>0$ such that

$$
\begin{aligned}
\left\|\sup _{(x, y) \in \mathbb{R}^{n} \times \mathbb{R}^{n} \otimes \mathbb{R}^{2 d}}\right\| \ell^{\alpha, \beta}(\cdot, x, y)-\ell^{\alpha_{m}, \beta_{m}}(\cdot, x, y)\left\|_{\mathbb{R}^{n}}\right\|_{\mathcal{H}^{2}(\bar{\Omega} \times[0, T] ; \mathbb{R})} \\
\quad \leq c\left(\left\|\alpha-\alpha_{m}\right\|_{\mathcal{H}^{2}\left(\bar{\Omega} \times[0, T] ; \mathbb{R}^{n}\right)}+\left\|\beta-\beta_{m}\right\|_{\mathcal{H}^{2}\left(\bar{\Omega} \times[0, T] ; \mathbb{R}^{n} \otimes \mathbb{R}^{2 d}\right)}\right)
\end{aligned}
$$

for all $m \in \mathbb{N}$. Hence, by the Markov inequality, for any $\epsilon>0$ then

$$
\lim _{m \rightarrow \infty} \overline{\mathbb{P}} \otimes \operatorname{Leb}\left(\sup _{(x, y) \in \mathbb{R}^{n} \times \mathbb{R}^{n} \otimes \mathbb{R}^{d}}\left\|\ell^{\alpha, \beta}(\cdot, x, y)-\ell^{\alpha_{m}, \beta_{m}}(\cdot, x, y)\right\|_{\mathbb{R}^{n}}>\epsilon\right)=0 .
$$

Therefore there exists a subset $S \in \overline{\mathcal{F}}_{T} \times \mathcal{B}([0, T])$ with $\overline{\mathbb{P}} \otimes \operatorname{Leb}(S)=T$ and a subsequence $\left\{m_{k} \subseteq\right.$ $\mathbb{N} \mid k \in \mathbb{N}\}$ such that

$$
\ell^{\alpha, \beta}(\bar{\omega}, t, x, y)=\lim _{k \rightarrow \infty} \ell^{\alpha_{m_{k}}, \beta_{m_{k}}}(\bar{\omega}, t, x, y)
$$


for all $(\bar{\omega}, t, x, y) \in S \times \mathbb{R}^{n} \times \mathbb{R}^{n} \otimes \mathbb{R}^{2 d}$. Note that

$$
\begin{aligned}
& I:=\left\{(\bar{\omega}, t, x, y) \mid \lim _{k \rightarrow \infty} \ell^{\alpha_{m_{k}}, \beta_{m_{k}}}(\bar{\omega}, t, x, y) \text { exists in } \mathbb{R}^{n}\right\} \\
& \quad=\left(\bigcup_{q \in \mathbb{Q}}\left(\left\{\liminf _{k \rightarrow \infty} \ell^{\alpha_{m_{k}}, \beta_{m_{k}}}<q\right\} \cap\left\{\limsup _{k \rightarrow \infty} \ell^{\alpha_{m_{k}}, \beta_{m_{k}}}>q\right\}\right)\right)^{c} \in \mathcal{P}(\overline{\mathbb{F}}) \times \mathcal{B}\left(\mathbb{R}^{n} \times \mathbb{R}^{n} \otimes \mathbb{R}^{2 d}\right) .
\end{aligned}
$$

Define $\bar{\ell}^{\alpha, \beta}: \bar{\Omega} \times[0, T] \times \mathbb{R}^{n} \times \mathbb{R}^{n} \otimes \mathbb{R}^{2 d} \rightarrow \mathbb{R}^{n}$ by

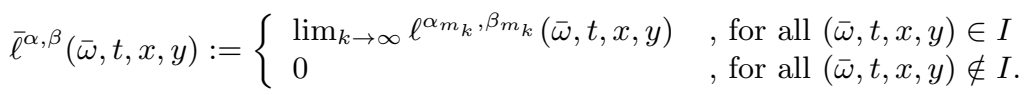

Thus $\bar{\ell}^{\alpha, \beta}$ is $\mathcal{P}(\overline{\mathbb{F}}) \times \mathcal{B}\left(\mathbb{R}^{n} \times \mathbb{R}^{n} \otimes \mathbb{R}^{2 d}\right)$-measurable, and from equation (16) then

$$
\ell^{\alpha, \beta}(\bar{\omega}, t, x, y)=\bar{\ell}^{\alpha, \beta}(\bar{\omega}, t, x, y)
$$

for all $(\bar{\omega}, t, x, y) \in S \times \mathbb{R}^{n} \times \mathbb{R}^{n} \otimes \mathbb{R}^{2 d}$. That is $\ell^{\alpha, \beta}$ is in the $\overline{\mathbb{P}} \otimes$ Leb-almost sure equivalence class of the $\mathcal{P}(\overline{\mathbb{F}}) \times \mathcal{B}\left(\mathbb{R}^{n} \times \mathbb{R}^{n} \otimes \mathbb{R}^{2 d}\right)$-measurable function $\bar{\ell}^{\alpha, \beta}$.

\section{Conclusion}

The main result of this paper is the SMP of Theorem 3.6, which provides necessary conditions for the existence of Nash equilibria in the SDG that we consider here. At the conceptual level, the main contribution of this work could be seen as the introduction of the notion of CMFBSDEs, and the representation, in the proof of Theorem 3.6, of part of the second form of quadratic-type term - that is a Peng-type adjoint representation (see [21]) with the adjoint processes defined as solutions to certain such CMFBSDEs. In the proof of Theorem 3.6, we used this representation to complete the calculation of the limit as $\epsilon \downarrow 0$ of the second form of quadratic-type term multiplied by $\epsilon^{-1}$. This is a different approach to that which is used in [5] to deal with the comparable type of term in their work. We propose that our approach to this matter is no more complicated than that in [5].

Moreover, it would appear that - in principle - our approach would be suited to further development in an attempt to calculate higher-than-first-order expansion terms, in this mean-field setting. Our approach to treating the second form of quadratic-type term gives a certain insight into its convergence properties. We suspect that, given appropriate conditions, this approach could be further developed (so as to include the various required elements that were clearly seen to be of the order $o(\epsilon)$, and as such were treated separately here) to yield a representation from which, possibly with the application of further Peng-type adjoint representations, higher-than-first-order expansion terms may be calculated. Such higher-order expansion terms would be of importance in the possible construction of higher-than-first-order SMPs (see below).

Possible directions for further research include the following.

- The application of our SMP to analyse example SDG problems of this type from, for example, mathematical finance. Such as, possible multi-agent, comparative performance based, optimal investment problems for asset price models with mean-field dynamics (note that an example of an asset price model with mean-field dynamics is considered, and the relating portfolio optimization problem addressed, in [3]). Moreover, the investigation of both the SMP result and its possible inferences on example problems, under particular information specifications - that is under specific examples of the individual agent information filtrations.

- The construction of a notion of a higher-than-first-order SMP, in the non-mean-field or mean-field settings. That is a form of SMP which gives further necessary conditions for an optimal control, or Nash equilibrium controls, by calculating the higher-than-first-order expansion terms for $\epsilon \downarrow 0$ of the difference in the performance between the assumed optimal control, or Nash equilibrium controls, and spike-variations of them-for perturbation controls that, also, satisfy the SMP equation of the previous order (for example, for a $n^{t h}$-order SMP where $n$ is the smallest element of $\{1+m / 2 \mid m \in \mathbb{N}\}$ such that the $n^{t h}$-order expansion terms are not null, then the considered perturbation controls will be taken as satisfying the original first-order SMP).

- To explore the question of whether mean-field SDGs, of the type considered here, can be connected, in any sense, with the notion of mean-field games from the work of, for example, [16]. 


\section{A Appendix}

Lemma A.1. Assume the same hypothesis, and notation as for Theorem 3.6. For each $p \in[1, \infty)$ there exists a constant $c>0$ such that

$$
\left\|\sup _{t \in[0, T]}\left|\zeta^{(r, \epsilon)}(t)\right|\right\|_{L^{p}(\Omega ; \mathbb{R})} \leq c \epsilon^{1 / 2}
$$

for all $r \in[0, T)$ and $\epsilon \in(0, T-r]$.

Define:

- for each $j \in\{1,2\}$ a family of $\mathcal{P}(\mathbb{F})$-measurable processes $\left\{\eta_{j}^{(r, \epsilon)}: \Omega \times[0, T] \rightarrow \mathbb{R} \mid r \in[0, T), \epsilon \in\right.$ $(0, T-r]\}$, for each $r \in[0, T)$ and $\epsilon \in(0, T-r]$, by

$$
\begin{aligned}
\eta_{j}^{(r, \epsilon)}(t):= & -\frac{1}{2} \partial^{(2)} \psi_{j}(t)+\int_{0}^{1} \int_{0}^{\lambda} \partial^{(2)} \psi_{j}\left(t, X^{*}(t)+\lambda^{\prime} \zeta^{(r, \epsilon)}(t),\right. \\
& \int_{\Omega} \phi_{\psi_{j}}\left(\omega^{\prime}, t, X^{*}\left(\omega^{\prime}, t\right)+\lambda^{\prime} \zeta^{(r, \epsilon)}\left(\omega^{\prime}, t\right), u^{*}\left(\omega^{\prime}, t\right)\right) \mathbb{P}\left(\mathrm{d} \omega^{\prime}\right), u^{*}(t), \zeta^{(r, \epsilon)}(t), \\
& \int_{\Omega} \partial \phi_{\psi_{j}}\left(\omega^{\prime}, t, X^{*}\left(\omega^{\prime}, t\right)+\lambda^{\prime} \zeta^{(r, \epsilon)}\left(\omega^{\prime}, t\right), u^{*}\left(\omega^{\prime}, t\right)\right) \zeta^{(r, \epsilon)}\left(\omega^{\prime}, t\right) \mathbb{P}\left(\mathrm{d} \omega^{\prime}\right), \\
& \left.\int_{\Omega} \partial^{(2)} \phi_{\psi_{j}}\left(\omega^{\prime}, t, X^{*}\left(\omega^{\prime}, t\right)+\lambda^{\prime} \zeta^{(r, \epsilon)}\left(\omega^{\prime}, t\right), u^{*}\left(\omega^{\prime}, t\right)\right) \zeta^{(r, \epsilon)}\left(\omega^{\prime}, t\right)^{2} \mathbb{P}\left(\mathrm{d} \omega^{\prime}\right)\right) \mathrm{d} \lambda^{\prime} \mathrm{d} \lambda
\end{aligned}
$$

for $\mathbb{P}$-almost all $\omega \in \Omega$, and uniformly for all $t \in[0, T]$, where $\psi_{1}:=b, \psi_{2}:=\sigma$.

- for each $i \in\{1, \ldots, N\}$, a family of $\mathcal{F}$-measurable random variables, $\left\{\eta_{3, i}^{(r, \epsilon)}: \Omega \rightarrow \mathbb{R} \mid r \in\right.$ $[0, T), \epsilon \in(0, T-r]\}$, for each $r \in[0, T)$ and $\epsilon \in(0, T-r]$, by

$$
\begin{aligned}
\eta_{3, i}^{(r, \epsilon)}:=-\frac{1}{2} \partial^{(2)} g_{i} & +\int_{0}^{1} \int_{0}^{\lambda} \partial^{(2)} g_{i}\left(X^{*}(T)+\lambda^{\prime} \zeta^{(r, \epsilon)}(T),\right. \\
& \int_{\Omega} \phi_{g_{i}}\left(\omega^{\prime}, X^{*}\left(\omega^{\prime}, T\right)+\lambda^{\prime} \zeta^{(r, \epsilon)}\left(\omega^{\prime}, T\right)\right) \mathbb{P}\left(\mathrm{d} \omega^{\prime}\right), \zeta^{(r, \epsilon)}(T), \\
& \int_{\Omega} \partial \phi_{g_{i}}\left(\omega^{\prime}, X^{*}\left(\omega^{\prime}, T\right)+\lambda^{\prime} \zeta^{(r, \epsilon)}\left(\omega^{\prime}, T\right)\right) \zeta^{(r, \epsilon)}\left(\omega^{\prime}, T\right) \mathbb{P}\left(\mathrm{d} \omega^{\prime}\right), \\
& \left.\int_{\Omega} \partial^{(2)} \phi_{g_{i}}\left(\omega^{\prime}, X^{*}\left(\omega^{\prime}, T\right)+\lambda^{\prime} \zeta^{(r, \epsilon)}\left(\omega^{\prime}, T\right)\right) \zeta^{(r, \epsilon)}\left(\omega^{\prime}, T\right)^{2} \mathbb{P}\left(\mathrm{d} \omega^{\prime}\right)\right) \mathrm{d} \lambda^{\prime} \mathrm{d} \lambda
\end{aligned}
$$

for $\mathbb{P}$-almost all $\omega \in \Omega$.

- for each $i \in\{1, \ldots, N\}$, a family of $\mathcal{P}(\mathbb{F})$-measurable processes $\left\{\eta_{4, i}^{(r, \epsilon)}: \Omega \times[0, T] \rightarrow \mathbb{R} \mid r \in\right.$ $[0, T), \epsilon \in(0, T-r]\}$, for each $r \in[0, T)$ and $\epsilon \in(0, T-r]$, by

$$
\begin{aligned}
\eta_{4, i}^{(r, \epsilon)}(t):= & -\frac{1}{2} \partial^{(2)} f_{i}(t)+\int_{0}^{1} \int_{0}^{\lambda} \partial^{(2)} f_{i}\left(t, X^{*}(t)+\lambda^{\prime} \zeta^{(r, \epsilon)}(t),\right. \\
& \int_{\Omega} \phi_{f_{i}}\left(\omega^{\prime}, t, X^{*}\left(\omega^{\prime}, t\right)+\lambda^{\prime} \zeta^{(r, \epsilon)}\left(\omega^{\prime}, t\right), u^{*}\left(\omega^{\prime}, t\right)\right) \mathbb{P}\left(\mathrm{d} \omega^{\prime}\right), u^{*}(t), \zeta^{(r, \epsilon)}(t), \\
& \int_{\Omega} \partial \phi_{f_{i}}\left(\omega^{\prime}, t, X^{*}\left(\omega^{\prime}, t\right)+\lambda^{\prime} \zeta^{(r, \epsilon)}\left(\omega^{\prime}, t\right), u^{*}\left(\omega^{\prime}, t\right)\right) \zeta^{(r, \epsilon)}\left(\omega^{\prime}, t\right) \mathbb{P}\left(\mathrm{d} \omega^{\prime}\right), \\
& \left.\int_{\Omega} \partial^{(2)} \phi_{f_{i}}\left(\omega^{\prime}, t, X^{*}\left(\omega^{\prime}, t\right)+\lambda^{\prime} \zeta^{(r, \epsilon)}\left(\omega^{\prime}, t\right), u^{*}\left(\omega^{\prime}, t\right)\right) \zeta^{(r, \epsilon)}\left(\omega^{\prime}, t\right)^{2} \mathbb{P}\left(\mathrm{d} \omega^{\prime}\right)\right) \mathrm{d} \lambda^{\prime} \mathrm{d} \lambda
\end{aligned}
$$

for $\mathbb{P}$-almost all $\omega \in \Omega$ and uniformly for all $t \in[0, T]$.

The following properties are satisfied:

- For each $r \in[0, T)$ and $\epsilon \in(0, T-r]$ then

$$
\begin{aligned}
\zeta^{(r, \epsilon)}(t)=\zeta^{(r, \epsilon)}(r+\epsilon)+\int_{r+\epsilon}^{t}(\partial b(s)+ & \left.\frac{1}{2} \partial^{(2)} b(s)+\eta_{1}^{(r, \epsilon)}(s)\right) \mathrm{d} s \\
& +\int_{r+\epsilon}^{t}\left(\partial \sigma(s)+\frac{1}{2} \partial^{(2)} \sigma(s)+\eta_{2}^{(r, \epsilon)}(s)\right) \mathrm{d} B(s)
\end{aligned}
$$

for $\mathbb{P}$-almost all $\omega \in \Omega$, and uniformly for all $t \in[r+\epsilon, T]$. 
- For each $i \in\{1, \ldots, N\}, r \in[0, T)$ and $\epsilon \in(0, T-r]$ then

$$
\begin{array}{r}
g_{i}\left(X^{(r, \epsilon)}(T), \int_{\Omega} \phi_{g_{i}}\left(\omega^{\prime}, X^{(r, \epsilon)}\left(\omega^{\prime}, T\right)\right) \mathbb{P}\left(\mathrm{d} \omega^{\prime}\right)\right)-g_{i}\left(X^{*}(T), \int_{\Omega} \phi_{g_{i}}\left(\omega^{\prime}, X^{*}\left(\omega^{\prime}, T\right)\right) \mathbb{P}\left(\mathrm{d} \omega^{\prime}\right)\right) \\
=\partial g_{i}+\frac{1}{2} \partial^{(2)} g_{i}+\eta_{3, i}^{(r, \epsilon)}
\end{array}
$$

for $\mathbb{P}$-almost all $\omega \in \Omega$.

- For each $i \in\{1, \ldots, N\}, r \in[0, T)$ and $\epsilon \in(0, T-r]$ then

$$
\delta^{(r, \epsilon)} f_{i}(t)=\partial f_{i}(t)+\frac{1}{2} \partial^{(2)} f_{i}(t)+\eta_{4, i}^{(r, \epsilon)}(t)
$$

for $\mathbb{P}$-almost all $\omega \in \Omega$, and uniformly for all $t \in[r+\epsilon, T]$.

- For each $p \in[1, \infty)$ there exists a constant $c>0$ such that

$$
\mathbb{E}_{\mathbb{P}}\left[\sup _{t \in[0, T]}\left|\eta_{1}^{(r, \epsilon)}(t)\right|^{p}+\sup _{t \in[0, T]}\left|\eta_{2}^{(r, \epsilon)}(t)\right|^{p}+\sum_{i=1}^{N}\left(\left|\eta_{3, i}^{(r, \epsilon)}\right|^{p}+\sup _{t \in[0, T]}\left|\eta_{4, i}^{(r, \epsilon)}(t)\right|^{p}\right)\right] \leq c \epsilon^{3 p / 2}
$$

for all $r \in[0, r)$ and $\epsilon \in(0, T-r]$.

Proof. Given Assumption 2.1 then this lemma may be established using the standard general argument for such types of results, that using: the Taylor expansion, the Burkholder-Davis-Gundy inequalities and the Gronwall inequality. Note that: a related result to that of equation (17) is given in equation (3.8) of Proposition 3.1 in [5]; related decompositions of the form in equations (18), (19) and (20) appear in [20] for a non-mean-field setting.

\section{Acknowledgements}

The author wishes to thank Agnès Sulem, Jean-Pierre Quadrat and Bernt Øksendal for helpful discussions and advice on this work and/or research leading to it. Also, the author is grateful to Boualem Djehiche for first bringing the work that became [5] to the author's attention, and for further helpful discussions.

Time spent by the author on this work or other research leading to it, includes two periods spent visiting the Centre of Mathematics for Applications (CMA) at the University of Oslo (once in 2009 and once in 2010). The author most gratefully acknowledges financial support from the CMA for these visits.

\section{References}

[1] D. Andersson and B. Djehiche. A Maximum Principle for SDEs of Mean-Field Type. Appl. Math. Optim., 63:341-356, 2011.

[2] A. Bensoussan. Lectures on stochastic control. In S. K. Mitter and A. Moro, editors, Nonlinear Filtering and Stochastic Control, volume 972 of Lecture Notes in Mathematics, pages 1-62. Springer Berlin / Heidelberg, 1982.

[3] V. S. Borkar and K. S. Kumar. McKean-Vlasov Limit in Portfolio Optimization. Stochastic Analysis and Applications, 28:884-906, 2010.

[4] M. Bossy and D. Talay. A stochastic particle method for the McKean-Vlasov and the Burgers equation. Mathematics of Computation, 66(217):157-192, 1997.

[5] R. Buckdahn, B. Djehiche, and J. Li. A General Stochastic Maximum Principle for SDEs of Mean-field Type. Appl. Math. Optim., 64:197-216, 2011.

[6] R. Buckdahn, B. Djehiche, J. Li, and S. Peng. Mean-field backward stochastic differential equations: a limit approach. The Annals of Probability, 37(4):1524-1565, 2009.

[7] R. Buckdahn, J. Li, and S. Peng. Mean-field backward stochastic differential equations and related partial differential equations. Stochastic Processes and their Applications, 119(10):31333154, 2009. 
[8] N. El Karoui, S. Peng, and M. C. Quenez. Backward stochastic differential equations in finance. Mathematical Finance, 7(1):1-71, 1997.

[9] O. Guéant, J.-M. Lasry, and P.-L. Lions. Mean Field Games and Applications. In R. A. Carmona, E. Cinlar, I. Ekeland, E. Jouini, J. A. Scheinkman, and N. Touzi, editors, Paris-Princeton Lectures on Mathematical Finance 2010, volume 2003 of Lecture Notes in Mathematics, pages 205-266. Springer Berlin / Heidelberg, 2011.

[10] J. J. A. Hosking. A stochastic maximum principle for a stochastic differential game of a mean-field type. HAL - INRIA, paperId: hal-00641090, version 1. URL: http://hal.inria.fr/hal-00641090/en/, November 2011.

[11] B. Jourdain, S. Méléard, and W. A. Woyczynski. Nonlinear SDEs driven by Lévy processes and related PDEs. Alea, 4:1-29, 2008.

[12] Y. M. Kabanov. On the Pontryagin maximum principle for SDEs with a Poisson-type driving noise. In Y. M. Kabanov, B. L. Rozovskii, and A. N. Shiryaev, editors, Statistics and control of stochastic processes. The Liptser Festschrift, pages 173-190. World Scientific Publishing Co., Inc., River Edge, New Jersey, 1997.

[13] K. Kuratowski and C. Ryll-Nardzewski. A General Theorem on Selectors. Bull. Acad. Polon. Sci. Sér. Sci. Math. Astronom. Phys., 13:397-403, 1965.

[14] J.-M. Lasry and P.-L. Lions. Jeux à champ moyen. I - Le cas stationnaire. C. R. Acad. Sci. Paris, Ser. I, 343:619-625, 2006.

[15] J.-M. Lasry and P.-L. Lions. Jeux à champ moyen. II - Horizon fini et contrôle optimal. C. R. Acad. Sci. Paris, Ser. I, 343:679-684, 2006.

[16] J.-M. Lasry and P.-L. Lions. Mean field games. Japan. J. Math., 2:229-260, 2007.

[17] S. Méléard. Asymptotic Behaviour of some interacting particle systems; McKean-Vlasov and Boltzmann models. In D. Talay and L. Tubaro, editors, Probabilistic Models for Nonlinear Partial Differential Equations, volume 1627 of Lecture Notes in Mathematics, pages 42-95. Springer Berlin / Heidelberg, 1996.

[18] T. Meyer-Brandis, B. Øksendal, and X. Y. Zhou. A mean-field stochastic maximum principle via Malliavin calculus. Preprint series Pure Mathematics, No. 10, Dept. of Math./CMA University of Oslo, 2008. Revised May 7, 2010. ISSN 0806-2439.

[19] L. Mou and J. Yong. A Variational Formula for Stochastic Controls and Some Applications. Pure Appl. Math. Q., 3(2):539-567, 2007.

[20] B. Øksendal and X. Y. Zhou. Riesz Representation, Malliavin calculus, and Stochastic Maximum Principle. Preprint of work in preparation, 21 July 2008.

[21] S. Peng. A general stochastic maximum principle for optimal control problems. SIAM J. Control and Optimization, 28(4):966-979, 1990. 